\title{
Multiobjective Output-Feedback Control via LMI Optimization
}

\author{
Carsten Scherer, Member, IEEE, Pascal Gahinet, Member, IEEE, and Mahmoud Chilali
}

\begin{abstract}
This paper presents an overview of a linear matrix inequality (LMI) approach to the multiobjective synthesis of linear output-feedback controllers. The design objectives can be a mix of $H_{\infty}$ performance, $H_{2}$ performance, passivity, asymptotic disturbance rejection, time-domain constraints, and constraints on the closed-loop pole location. In addition, these objectives can be specified on different channels of the closed-loop system. When all objectives are formulated in terms of a common Lyapunov function, controller design amounts to solving a system of linear matrix inequalities. The validity of this approach is illustrated by a realistic design example.
\end{abstract}

Index Terms-Controller parameter change, linear matrix inequalities, Lyapunov shaping paradigm, multichannel multiobjective control.

\section{INTRODUCTION}

$\mathbf{L}$ INEAR matrix inequalities (LMI's) have emerged as a powerful formulation and design technique for a variety of linear control problems [9]. Since solving LMI's is a convex optimization problem, such formulations offer a numerically tractable means of attacking problems that lack an analytical solution. In addition, efficient interior-point algorithms are now available to solve the generic LMI problems with a polynomial-time worst-case complexity [8], [18], [25], [26], [32]. Consequently, reducing a control design problem to an LMI can be considered as a practical solution to this problem [9].

General multiobjective control problems are difficult and remain mostly open to this date. By multiobjective control, we refer to synthesis problems with a mix of time- and frequencydomain specifications ranging from $\mathrm{H}_{2}$ and $H_{\infty}$ performance to regional pole placement, asymptotic tracking or regulation, and settling time or saturation constraints.

For the multiobjective $\mathrm{H}_{2} / \mathrm{H}_{\infty}$ problem, it has been proposed to specify the closed-loop objectives in terms of a common Lyapunov function [7], [22]. This still guarantees the desired specifications at the expense of conservatism. As a benefit, controller design can be reduced to a convex optimization problem [22]. The same technique has proved valuable in arriving at design procedures for various state-feedback

Manuscript received December 8, 1995; revised January 20, 1997. Recommended by Associate Editor, A. Vicino.

C. Scherer is with the Mechanical Engineering Systems and Control Group, Delft University of Technology, 2628 CD Delft, The Netherlands (e-mail: c.scherer@wbmt.tudelft.nl).

P. Gahinet is with The MathWorks Inc., Natick, MA 01760-1500 USA.

M. Chilali is with INRIA Rocquencourt, Domaine de Voluceau, BP 105, 78153 Le Chesnay Cedex, France.

Publisher Item Identifier S 0018-9286(97)05069-1. control problems [5], [9], [11], [22]. In [29], a complete LMI treatment of the general mixed $H_{2} / H_{\infty}$ output-feedback synthesis problem was presented. This work eventually lead to realizing that output-feedback problems could be "linearized" by a mere change of controller variables, much like in the state-feedback case [11], [30]. While this change of variable is more sophisticated than its state-feedback counterpart, it offers an equally systematic means of turning output-feedback specifications into LMI's. We would like to stress that similar ideas emerged in the other independent works [23], [24].

The main purpose of this paper is to give a fairly complete overview of the design technique that guarantees the desired closed-loop specification in terms of a single Lyapunov function. The objectives addressed here include $\mathrm{H}_{2}$ and $H_{\infty}$ performance, passivity, peak output amplitude, peak-topeak gain, nominal and robust regulation, and regional pole placement. While most of these results are easily obtained by applying the controller parameter transformation proposed in [11], [23], and [30], the extension to nominal regulation requires a modification that is new and reveals the potential to address an even larger class of problems.

The paper is organized as follows. Section II gives the problem statement and motivations. Section III reviews the various specifications and objectives that can be formulated in terms of LMI's. Section IV discusses the design methodology and its interpretation as a "Lyapunov shaping" paradigm. It also defines the critical change of controller variables that allows us to linearize the problem and turns it into a set of LMI's. Section V lists the resulting LMI constraints for each individual specification and discusses how they can be combined to solve various multiobjective problems. Section VI comments on reduced-order controller design, and Section VII illustrates this approach on a realistic design example.

The notation is fairly standard. The compact notation

$$
\left[\begin{array}{l|l}
A & B \\
\hline C & D
\end{array}\right]
$$

is used to denote the transfer function $G(s)=D+C(s I-$ $A)^{-1} B$.

\section{Problem Statement and Motivations}

This paper deals with multiobjective output-feedback synthesis for multi-input/multi-output (MIMO) linear timeinvariant (LTI) systems. This section gives a formal statement of the problem and defines the relevant notation. The LTI 
plant is given by the state-space equations

$$
P\left\{\begin{array}{l}
\dot{x}=A x+B_{w} w+B u \\
z=C_{z} x+D_{z w} w+D_{z} u \\
y=C x+D_{w} w
\end{array}\right.
$$

where $u \in \boldsymbol{R}^{n_{u}}$ is the control input, $w$ is a vector of exogenous inputs (such as reference signals, disturbance signals, sensor noise), $y \in \boldsymbol{R}^{n_{y}}$ is the measured output, and $z$ is a vector of output signals related to the performance of the control system.

Let $T$ denote the closed-loop transfer functions from $w$ to $z$ for some dynamical output-feedback control law $u=K y$. Our goal is to compute a dynamical output-feedback controller

$$
K\left\{\begin{array}{l}
\dot{\zeta}=A_{K} \zeta+B_{K} y \\
u=C_{K} \zeta+D_{K} y
\end{array}\right.
$$

that meets various specifications on the closed-loop behavior. Typically, these specifications are defined for particular channels or combinations of channels. More precisely, each specification or objective is formulated relative to some closedloop transfer function of the form

$$
T_{j}=L_{j} T R_{j}
$$

where the matrices $L_{j}, R_{j}$ select the appropriate input/output (I/O) channels or channel combinations. Unlike previous work [13], [22], our approach does not require that the selected input or output channels are the same for all objectives. Rather, the multiobjective problem considered here is intrinsically multichannel.

The specifications and objectives under consideration include $H_{\infty}$ performance, $\mathrm{H}_{2}$ performance, dissipativeness, time-domain constraints (peak amplitude, overshoot, settling time), and regulation. Additional regional constraints on the closed-loop poles can also be imposed. The motivations for using such a mix of performance measures are as follows.

- The $H_{\infty}$ performance is convenient to enforce robustness to model uncertainty and to express frequency-domain specifications such as bandwidth, low-frequency gain, and roll-off.

- The $H_{2}$ performance is useful to handle stochastic aspects such as measurement noise and random disturbance.

- Passivity requirements appear in specific control systems such as flexible structures and circuits.

- Time-domain constraints are useful to tune the transient response and peak amplitudes such as the peak of the impulse response, the overshoot of the step response, or the peak control input.

- It is often desirable to enforce some minimum decay rate or closed-loop damping via regional pole assignment [11]. In addition, pole constraints are useful to avoid fast dynamics and high-frequency gain in the controller, which in turn facilitate its digital implementation.

- A general goal is the asymptotic rejection of disturbance or tracking of reference signals that are generated by a known model (integral control).
In summation, multiobjective design allows for more flexible and accurate specification of the desirable closed-loop behavior.

Henceforth, all specifications and objectives are expressed in terms of the transfer function $T_{j}$, keeping in mind that $T_{j}$ refers to any particular I/O channel in the closed-loop mapping. Since our approach is state-space based, we first provide a statespace realization for $T_{j}$ and introduce some useful shorthand notation. With the plant $P$ and controller $K$ defined as above, the closed-loop system admits the realization

$$
T\left\{\begin{aligned}
\dot{x}_{c l} & =\mathcal{A} x_{c l}+\mathcal{B} w \\
z & =\mathcal{C} x_{c l}+\mathcal{D} w
\end{aligned}\right.
$$

where

$$
\begin{aligned}
& \left(\begin{array}{l|l}
\mathcal{A} & \mathcal{B} \\
\hline \mathcal{C} & \mathcal{D}
\end{array}\right) \\
& \quad=\left(\begin{array}{ccc}
A+B D_{K} C & B C_{K} & B_{w}+B D_{K} D_{w} \\
B_{K} C & A_{K} & B_{K} D_{w} \\
\hline C_{z}+D_{z} D_{K} C & D_{z} C_{K} & D_{z w}+D_{z} D_{K} D_{w}
\end{array}\right) .
\end{aligned}
$$

With

$$
\begin{aligned}
& B_{j}:=B_{w} R_{j}, \quad C_{j}:=L_{j} C_{z}, \quad D_{j}:=L_{j} D_{z w} R_{j} \\
& E_{j}:=L_{j} D_{z}, \quad F_{j}:=D_{w} R_{j}
\end{aligned}
$$

it is readily verified that a realization of $T_{j}(s)=L_{j} T(s) R_{j}$ is given by

$$
\begin{aligned}
\left(\begin{array}{c|c}
\mathcal{A} & \mathcal{B}_{j} \\
\hline \mathcal{C}_{j} & \mathcal{D}_{j}
\end{array}\right) \\
:=\left(\begin{array}{c|c}
\mathcal{A} & \mathcal{B} R_{j} \\
\hline L_{j} \mathcal{C} & L_{j} \mathcal{D} R_{j}
\end{array}\right) \\
=\left(\begin{array}{cc|c}
A+B D_{K} C & B C_{K} & B_{j}+B D_{K} F_{j} \\
B_{K} C & A_{K} & B_{K} F_{j} \\
\hline C_{j}+E_{j} D_{K} C & E_{j} C_{K} & D_{j}+E_{j} D_{K} F_{j}
\end{array}\right) .
\end{aligned}
$$

Note that $T_{j}(s)$ is nothing but the transfer function from $w_{j}$ to $z_{j}$ if specifying the input and output signals in (4) as $w=R_{j} w_{j}$ and $z_{j}=L_{j} z$.

\section{LMI Formulation OF THE DESIGN SPECIFICATIONS}

This section gives an overview of the various closed-loop specifications and objectives that can be captured in the LMI framework. All LMI characterizations listed below have the following common origin: let $\mathcal{A}$ and $x_{c l}$ denote the closed-loop state matrix and state vector, respectively. Since the controller has to be internally stabilizing, the closed-loop system must admit a quadratic Lyapunov function

$$
V\left(x_{c l}\right)=x_{c l}^{T} \mathcal{P} x_{c l}, \quad \mathcal{P}>0
$$

such that

$$
\mathcal{A}^{T} \mathcal{P}+\mathcal{P} \mathcal{A}<0
$$

The LMI approach consists of expressing each control specification or objective as an additional constraint on admissible 
closed-loop Lyapunov functions satisfying (6) and (7). In the multichannel spirit, we consider specifications on a generic closed-loop transfer function $T_{j}(s)=L_{j} T(s) R_{j}$, whose realization is given in (5). The corresponding LMI constraints are therefore formulated in terms of the state-space matrices $\mathcal{A}, \mathcal{B}_{j}, \mathcal{C}_{j}, \mathcal{D}_{j}$

Remark 1: Given a quadratic function $Q\left(w_{j}, z_{j}\right)$, we use the shorthand notation $Q\left(w_{j}, z_{j}\right) \ll 0$ to denote the existence of $\epsilon>0$ such that $Q\left(w_{j}, z_{j}\right) \leq-\epsilon w_{j}^{T} w_{j}$ for all $w_{j}$. Similarly

$$
\int_{0}^{T} Q\left(w_{j}(t), z_{j}(t)\right) d t \ll 0
$$

means that

$$
\int_{0}^{T} Q\left(w_{j}(t), z_{j}(t)\right) d t \leq-\epsilon \int_{0}^{T} w_{j}(t)^{T} w_{j}(t) d t
$$

holds for all square-integrable inputs $w_{j}($.$) and some fixed$ $\epsilon>0$.

\section{A. $H_{\infty}$ Performance}

Let $\left\|T_{j}\right\|_{\infty}$ denote the $H_{\infty}$ norm of $T_{j}$, that is, its largest gain across frequency in the singular value norm [15]. The $H_{\infty}$ norm measures the system input-output gain for finite energy or finite rms input signals. The constraint $\left\|T_{j}\right\|_{\infty}<\gamma$ can be interpreted as a disturbance rejection performance. This constraint is also useful to enforce robust stability. Specifically, it guarantees that the closed-loop system remains stable for all perturbations $w=R_{j} \Delta L_{j} z$, with $\Delta$ having incremental gain not larger than $1 / \gamma$.

By virtue of the Bounded Real Lemma [4], [28], $\mathcal{A}$ is stable and the $H_{\infty}$ norm of $T_{j}$ is smaller than $\gamma$ if and only if there exists a symmetric $\mathcal{P}$ with

$$
\left(\begin{array}{ccc}
\mathcal{A}^{T} \mathcal{P}+\mathcal{P} \mathcal{A} & \mathcal{P} \mathcal{B}_{j} & \mathcal{C}_{j}^{T} \\
\mathcal{B}_{j}^{T} \mathcal{P} & -\gamma I & \mathcal{D}_{j}^{T} \\
\mathcal{C}_{j} & \mathcal{D}_{j} & -\gamma I
\end{array}\right)<0, \quad \quad \mathcal{P}>0
$$

\section{B. General Quadratic Constraints}

Given fixed matrices $U_{j} \geq 0, V_{j}=V_{j}^{T}$, and $W_{j}$, the previous characterization extends to more general quadratic constraints on $w_{j}(),. z_{j}($.$) of the form$

$$
\int_{0}^{T}\left(\begin{array}{c}
z_{j}(t) \\
w_{j}(t)
\end{array}\right)^{T}\left(\begin{array}{cc}
U_{j} & W_{j} \\
W_{j}^{T} & V_{j}
\end{array}\right)\left(\begin{array}{c}
z_{j}(t) \\
w_{j}(t)
\end{array}\right) d t \ll 0
$$

for $x_{c l}(0)=0$ and all $T \geq 0, w_{j} \in L_{2}$ (with $\ll$ defined in Remark 1).

Important special cases include:

- the $H_{\infty}$ constraint $\left\|T_{j}\right\|_{\infty}<\gamma$ which corresponds to $U_{j}=(1 / \gamma) I, V_{j}=-\gamma I, W_{j}=0$

- the strict passivity constraint $T_{j}(i \omega)+T_{j}(i \omega)^{*}>0$ for all $\omega \in \mathbf{R} \cup\{\infty\}$ which corresponds to $U_{j}=0, V_{j}=0$, $W_{j}=-I$;

- sector constraints: with $U_{j}=I, V_{j}=-\alpha \beta I, W_{j}=$ $-\frac{1}{2}(\alpha+\beta) I,(9)$ reads

$$
\int_{0}^{T}\left(z_{j}(t)-\alpha w_{j}(t)\right)^{T}\left(z_{j}(t)-\beta w_{j}(t)\right) d t \ll 0
$$

which is to say that the mapping $T_{j}=L_{j} T R_{j}$ lies in the sector $\{\alpha, \beta\}$.

Suppose that $\mathcal{P}>0$ and

$$
\begin{aligned}
\left(\begin{array}{cc}
\mathcal{A}^{T} \mathcal{P}+\mathcal{P} \mathcal{A} & \mathcal{P} \mathcal{B}_{j} \\
\mathcal{B}_{j}^{T} \mathcal{P} & 0
\end{array}\right) & +\left(\begin{array}{cc}
\mathcal{C}_{j} & \mathcal{D}_{j} \\
0 & I
\end{array}\right)^{T} \\
& \cdot\left(\begin{array}{cc}
U_{j} & W_{j} \\
W_{j}^{T} & V_{j}
\end{array}\right)\left(\begin{array}{cc}
\mathcal{C}_{j} & \mathcal{D}_{j} \\
0 & I
\end{array}\right)<0 .
\end{aligned}
$$

Recalling that $z_{j}=\mathcal{C}_{j} x_{c l}+\mathcal{D}_{j} w_{j}$ and that

$$
\frac{d}{d t} V\left(x_{c l}(t)\right)=\left(\begin{array}{l}
x_{c l}(t) \\
w_{j}(t)
\end{array}\right)^{T}\left(\begin{array}{cc}
\mathcal{A}^{T} \mathcal{P}+\mathcal{P} \mathcal{A} & \mathcal{P} \mathcal{B}_{j} \\
\mathcal{B}_{j}^{T} \mathcal{P} & 0
\end{array}\right)\left(\begin{array}{l}
x_{c l}(t) \\
w_{j}(t)
\end{array}\right)
$$

for $V$ defined in (6), we infer from (10) that

$$
\frac{d}{d t} V\left(x_{c l}(t)\right)+\left(\begin{array}{c}
z_{j}(t) \\
w_{j}(t)
\end{array}\right)^{T}\left(\begin{array}{cc}
U_{j} & W_{j} \\
W_{j}^{T} & V_{j}
\end{array}\right)\left(\begin{array}{c}
z_{j}(t) \\
w_{j}(t)
\end{array}\right) \ll 0 .
$$

Integration from $t=0$ to $t=T$ then yields

$$
\begin{aligned}
x_{c l}^{T}(T) \mathcal{P} x_{c l}(T) & +\int_{0}^{T}\left(\begin{array}{c}
z_{j}(t) \\
w_{j}(t)
\end{array}\right)^{T} \\
& \cdot\left(\begin{array}{cc}
U_{j} & W_{j} \\
W_{j}^{T} & V_{j}
\end{array}\right)\left(\begin{array}{c}
z_{j}(t) \\
w_{j}(t)
\end{array}\right) d t \ll 0
\end{aligned}
$$

and (9) follows by observing that $x_{c l}(T) \mathcal{P} x_{c l}(T) \geq 0$.

Conversely, (9) implies that there exists an $\epsilon>0$ with

$$
\begin{aligned}
\int_{0}^{T}\left(\begin{array}{c}
z_{j}(t) \\
w_{j}(t)
\end{array}\right)^{T}\left(\begin{array}{cc}
U_{j} & W_{j} \\
W_{j}^{T} & V_{j}
\end{array}\right)\left(\begin{array}{c}
z_{j}(t) \\
w_{j}(t)
\end{array}\right) \\
+\left(\begin{array}{l}
x_{c l}(t) \\
w_{j}(t)
\end{array}\right)^{T}\left(\begin{array}{cc}
\epsilon I & 0 \\
0 & \epsilon I
\end{array}\right)\left(\begin{array}{l}
x_{c l}(t) \\
w_{j}(t)
\end{array}\right) d t \leq 0 .
\end{aligned}
$$

By standard results from indefinite linear-quadratic (LQ) theory [33], we conclude that there exists a symmetric $\mathcal{P}$ satisfying

$$
\begin{aligned}
\left(\begin{array}{cc}
\mathcal{A}^{T} \mathcal{P}+\mathcal{P} \mathcal{A} & \mathcal{P} \mathcal{B}_{j} \\
\mathcal{B}_{j}^{T} \mathcal{P} & 0
\end{array}\right) & +\left(\begin{array}{cc}
\mathcal{C}_{j} & \mathcal{D}_{j} \\
0 & I
\end{array}\right)^{T} \\
& \cdot\left(\begin{array}{cc}
U_{j} & W_{j} \\
W_{j}^{T} & V_{j}
\end{array}\right)\left(\begin{array}{cc}
\mathcal{C}_{j} & \mathcal{D}_{j} \\
0 & I
\end{array}\right)+\epsilon I \leq 0 .
\end{aligned}
$$

(The controllability hypothesis in [33] can be weakened to stabilizability by a perturbation argument.) This implies (10). In addition, the left-upper block of (10) reads $\mathcal{A}^{T} \mathcal{P}+\mathcal{P} \mathcal{A}+$ $\mathcal{C}_{j}^{T} U_{j} \mathcal{C}_{j}<0$, which implies $\mathcal{P}>0$, since $\mathcal{A}$ is stable and $U_{j} \geq 0$.

Hence, we have proved that the existence of a positive definite solution of (10) is necessary and sufficient for $\mathcal{A}$ to be stable and (9) to hold.

For the purpose of synthesis we need to rewrite (10). We factorize $U_{j} \geq 0$ as

$$
U_{j}=Q_{j} \Sigma_{j}^{-1} Q_{j}^{T}, \quad \Sigma_{j}>0 .
$$

Then (10) is equivalent to

$$
\begin{aligned}
& \left(\begin{array}{cc}
\mathcal{A}^{T} \mathcal{P}+\mathcal{P} \mathcal{A} & \mathcal{P} \mathcal{B}_{j}+\mathcal{C}_{j}^{T} W_{j} \\
\mathcal{B}_{j}^{T} \mathcal{P}+W_{j}^{T} \mathcal{C}_{j} & \mathcal{D}_{j}^{T} W_{j}+W_{j}^{T} \mathcal{D}_{j}+V_{j}
\end{array}\right) \\
& +\left(\begin{array}{c}
\mathcal{C}_{j}^{T} \\
\mathcal{D}_{j}^{T}
\end{array}\right) Q_{j} \Sigma_{j}^{-1} Q_{j}^{T}\left(\mathcal{C}_{j} \quad \mathcal{D}_{j}\right)<0 .
\end{aligned}
$$


A Schur complement argument leads to the final analysis LMI's

$$
\left(\begin{array}{ccc}
\mathcal{A}^{T} \mathcal{P}+\mathcal{P} \mathcal{A} & \mathcal{P} \mathcal{B}_{j}+\mathcal{C}_{j}^{T} W_{j} & \mathcal{\mathcal { P }}>0 \\
\mathcal{B}_{j}^{T} \mathcal{P}+W_{j}^{T} \mathcal{C}_{j} & W_{j}^{T} \mathcal{D}_{j}+\mathcal{D}_{j}^{T} W_{j}+V_{j} & \mathcal{D}_{j}^{T} Q_{j} \\
Q_{j}^{T} \mathcal{C}_{j} & Q_{j}^{T} \mathcal{D}_{j} & -\Sigma_{j}
\end{array}\right)^{-}<0
$$

for general quadratic performance.

\section{C. $\mathrm{H}_{2}$ Performance}

Assume $\mathcal{A}$ is stable and $\mathcal{D}_{j}=0$. The $H_{2}$ norm of $T_{j}$ is defined by

$$
\left\|T_{j}\right\|_{2}^{2}:=\frac{1}{2 \pi} \int_{-\infty}^{+\infty} \operatorname{Tr}\left(T_{j}(i \omega)^{H} T_{j}(i \omega)\right) d \omega
$$

and corresponds to the asymptotic variance of the output $z_{j}=L_{j} z$ when the system is driven by $w=R_{j} w_{j}$ with white noise $w_{j}$. It is well known that this norm can be computed as $\left\|T_{j}\right\|_{2}^{2}=\operatorname{Tr}\left(\mathcal{C}_{j} \mathcal{S}_{0} \mathcal{C}_{j}^{T}\right)$, where $\mathcal{S}_{0}$ solves the Lyapunov equation

$$
\mathcal{A S}_{0}+\mathcal{S}_{0} \mathcal{A}^{T}+\mathcal{B}_{j} \mathcal{B}_{j}^{T}=0 .
$$

Since $\mathcal{S}_{0}<\mathcal{S}$ for any $\mathcal{S}$ satisfying

$$
\mathcal{A S}+\mathcal{S} \mathcal{A}^{T}+\mathcal{B}_{j} \mathcal{B}_{j}^{T}<0
$$

it is readily verified that $\left\|T_{j}\right\|_{2}^{2}<\nu$ if and only if there exists $\mathcal{S}>0$ satisfying (13) and $\operatorname{Tr}\left(\mathcal{C}_{j} \mathcal{S C}_{j}^{T}\right)<\nu$. With an auxiliary parameter $Q$, we obtain the following analysis result: $\mathcal{A}$ is stable and $\left\|T_{j}\right\|_{2}^{2}\left\langle\nu\right.$ iff there exist symmetric $\mathcal{P}:=\mathcal{S}^{-1}$ and $Q$ such that

$$
\begin{aligned}
\left(\begin{array}{cc}
\mathcal{A}^{T} \mathcal{P}+\mathcal{P} \mathcal{A} & \mathcal{P} \mathcal{B}_{j} \\
\mathcal{B}_{j}^{T} \mathcal{P} & -I
\end{array}\right)<0 & \\
& \left(\begin{array}{cc}
\mathcal{P} & \mathcal{C}_{j}^{T} \\
\mathcal{C}_{j} & Q
\end{array}\right)>0 \\
& \operatorname{Tr}(Q)<\nu, \quad \mathcal{D}_{j}=0 .
\end{aligned}
$$

\section{Generalized $\mathrm{H}_{2}$ Performance}

The $H_{\infty}$ norm considered in Section III-A gives the system gain when both the input and the output are measured in the energy or $L_{2}$ norm. Rather than bounding the output energy, it may be desirable to keep the peak amplitude of the output $z_{j}$ below a certain level, e.g., to avoid actuator saturations. If the input $w_{j}$ is still quantified by its energy, this leads to considering the so-called generalized $\mathrm{H}_{2}$-norm defined by

$$
\begin{aligned}
\left\|T_{j}\right\|_{g}:=\sup & \left\{\left\|z_{j}(T)\right\|: x_{c l}(0)=0,\right. \\
& \left.T \geq 0, \int_{0}^{T}\left\|w_{j}(t)\right\|^{2} d t \leq 1\right\} .
\end{aligned}
$$

This measures the peak amplitude of the output signal $z_{j}(t)$ over all unit-energy inputs $w_{j}(t)$.
Suppose that there exists a symmetric matrix $\mathcal{P}$ satisfying

$$
\begin{aligned}
\left(\begin{array}{cc}
\mathcal{A}^{T} \mathcal{P}+\mathcal{P} \mathcal{A} & \mathcal{P} \mathcal{B}_{j} \\
\mathcal{B}_{j}^{T} \mathcal{P} & -I
\end{array}\right)<0 & \\
& \left(\begin{array}{cc}
\mathcal{P} & \mathcal{C} \\
\mathcal{C}_{j} & \alpha I
\end{array}\right)>0, \quad \mathcal{D}_{j}=0
\end{aligned}
$$

The first inequality ensures that $(d / d t) V\left(x_{c l}(t)\right)-$ $w_{j}(t)^{T} w_{j}(t) \ll 0$, which yields after integration

$$
V\left(x_{c l}(T)\right) \ll \int_{0}^{T} w_{j}(t)^{T} w_{j}(t) d t .
$$

Meanwhile, the second inequality implies that $(1 / \alpha) \mathcal{C}_{j}^{T} \mathcal{C}_{j}<$ $\mathcal{P}$, and thus $z_{j}(T)^{T} z_{j}(T) \leq \alpha V\left(x_{c l}(T)\right)$. Combining these two inequalities leads to

$$
z_{j}(T)^{T} z_{j}(T) \ll \alpha \int_{0}^{T} w_{j}(t)^{T} w_{j}(t) d t
$$

for all $T \geq 0$, whence $\left\|T_{j}\right\|_{g}^{2}<\alpha$. It is not difficult to show that the solvability of (16) is also necessary for this norm bound to hold [27].

\section{E. Peak Impulse Response and Settling Time}

Suppose that $\mathcal{D}_{j}=0$ and that $T_{j}(s)$ is single input. Then the impulse response $z_{j}(t)$ coincides with the output of the system

$$
\left\{\begin{aligned}
\dot{x}_{c l} & =\mathcal{A} x_{c l}, \quad x_{c l}(0)=\mathcal{B}_{j} \\
z_{j} & =\mathcal{C}_{j} x_{c l} .
\end{aligned}\right.
$$

Following [9], a sufficient condition to guarantee that $\left\|z_{j}(t)\right\|<\xi$ for all $t \geq 0$ is the existence of a symmetric $\mathcal{P}$ such that

$$
\begin{array}{ll}
\mathcal{A}^{T} \mathcal{P}+\mathcal{P} \mathcal{A}<0, & \left(\begin{array}{cc}
\mathcal{P} & \mathcal{P} \mathcal{B}_{j} \\
\mathcal{B}_{j}^{T} \mathcal{P} & \xi I
\end{array}\right)>0 \\
\left(\begin{array}{cc}
\mathcal{P} & \mathcal{C}_{j}^{T} \\
\mathcal{C}_{j} & \xi I
\end{array}\right)>0, \quad \mathcal{D}_{j}=0 .
\end{array}
$$

Indeed, the inequalities respectively ensure that, for all $t \geq 0$

$$
\begin{aligned}
x_{c l}^{T}(t) \mathcal{P} x_{c l}(t) & \leq \mathcal{B}_{j}^{T} \mathcal{P} \mathcal{B}_{j}, \quad \quad \mathcal{B}_{j}^{T} \mathcal{P} \mathcal{B}_{j}<\xi \\
z_{j}^{T}(t) z_{j}(t) & \leq \xi x_{c l}^{T}(t) \mathcal{P} x_{c l}(t)
\end{aligned}
$$

and the bound $\left\|z_{j}(t)\right\|<\xi$ readily follows. Note that unlike most LMI characterizations given in this section, the LMI conditions (17) are only sufficient and can prove fairly conservative in some cases.

If replacing the first inequality in (17) by $\mathcal{A}^{T} \mathcal{P}+\mathcal{P} \mathcal{A}+$ $2 \alpha \mathcal{P}<0$, the bound on $\left\|z_{j}(t)\right\|$ can be improved to $\left\|z_{j}(t)\right\|<$ $\xi e^{-\alpha t}$. This can be used to impose an upper bound on the settling time through the appropriate choice of $\alpha>0$ [9].

\section{F. Bounds on the Peak-to-Peak Gain}

The generalized $\mathrm{H}_{2}$ norm measures the peak amplitude of the output over unit-energy inputs. Suppose, instead, that the input signal $w_{j}$ is only bounded in amplitude. To bound the 
peak amplitude of $z_{j}$, we then need to consider the so-called peak-to-peak gain of $T_{j}$ defined by

$$
\begin{gathered}
\left\|T_{j}\right\|_{\text {peak }}:=\sup \left\{\left\|z_{j}(T)\right\|: x_{c l}(0)=0, T \geq 0,\right. \\
\|w(t)\| \leq 1 \text { for } t \geq 0\} .
\end{gathered}
$$

This measures the peak norm of the output signal $z_{j}(t)$ for inputs $w_{j}(t)$ whose amplitude do not exceed one. Note that $\left\|T_{j}\right\|_{\infty} \leq\left\|T_{j}\right\|_{\text {peak }}$ as is easily seen by considering a sinusoidal input with frequency $\omega$ such that $\sigma_{\max }\left(T_{j}(j \omega)\right)=$ $\left\|T_{j}\right\|_{\infty}$.

To date there is no exact characterization of the peak-topeak norm in the LMI framework. However, it is possible to derive upper bounds for $\left\|T_{j}\right\|_{\text {peak }}$ along the lines of [3] and [9]. Suppose that $\mathcal{P}, \lambda, \mu$, and $\zeta$ satisfy

$$
\begin{gathered}
\left(\begin{array}{cc}
\mathcal{A}^{T} \mathcal{P}+\mathcal{P} \mathcal{A}+\lambda \mathcal{P} & \mathcal{P} \mathcal{B}_{j} \\
\mathcal{B}_{j}^{T} \mathcal{P} & -\mu I
\end{array}\right)<0 \\
\left(\begin{array}{ccc}
\lambda \mathcal{P} & 0 & \mathcal{C}_{j}^{T} \\
0 & (\zeta-\mu) I & \mathcal{D}_{j}^{T} \\
\mathcal{C}_{j} & \mathcal{D}_{j} & \zeta I
\end{array}\right)>0 .
\end{gathered}
$$

Choose any $w_{j}($.$) with w_{j}(t)^{T} w_{j}(t) \leq 1$ for $t \geq 0$. The second inequality implies that

$$
\frac{d}{d t} V\left(x_{c l}(t)\right)+\lambda V\left(x_{c l}(t)\right)-\mu w_{j}(t)^{T} w_{j}(t) \leq 0 .
$$

Hence $(d / d t) V\left(x_{c l}(t)\right) \leq 0$ holds whenever $V\left(x_{c l}(t)\right) \geq \mu / \lambda$. Since $V\left(x_{c l}(0)\right)=0$, this shows that $V\left(x_{c l}(t)\right)$ cannot exceed the value $\mu / \lambda$

$$
V\left(x_{c l}(t)\right) \leq \frac{\mu}{\lambda}, \quad \text { for } t \geq 0 .
$$

To derive a bound on $\left\|T_{j}\right\|_{\text {peak }}$, it now suffices to bound $z_{j}(t)$ in terms of $V\left(x_{c l}(t)\right)$ and $w_{j}(t)$, which is achieved through the third inequality in (18). Indeed, this inequality gives

$$
\frac{1}{\zeta}\left(\begin{array}{l}
\mathcal{C}_{j}^{T} \\
\mathcal{D}_{j}^{T}
\end{array}\right)\left(\mathcal{C}_{j} \quad \mathcal{D}_{j}\right)<\left(\begin{array}{cc}
\lambda \mathcal{P} & 0 \\
0 & (\zeta-\mu) I
\end{array}\right)
$$

and thus

$$
\begin{aligned}
z_{j}(t)^{T} z_{j}(t) & \ll \zeta\left(\lambda V\left(x_{c l}(t)\right)+(\zeta-\mu) w_{j}(t)^{T} w_{j}(t)\right) \\
& \leq \zeta\left(\lambda V\left(x_{c l}(t)\right)+(\zeta-\mu)\right) .
\end{aligned}
$$

Recalling from (20) that $\lambda V\left(x_{c l}(t)\right)-\mu \leq 0$, this yields $z_{j}(t)^{T} z_{j}(t) \ll \zeta^{2}$. Summing up, (18) secures the stability of $\mathcal{A}$ and the bound

$$
\left\|T_{j}\right\|_{\text {peak }}<\zeta \text {. }
$$

Note that (18) is only linear if fixing $\lambda$. Finding the best bound guaranteed by (18) hence requires performing a linesearch over $\lambda>0$. The implications for synthesis are clarified in Section V-E. For the purpose of the analysis, we observe that we can confine the search to $\lambda \in(0,-2 \max \operatorname{Re}(\sigma(\mathcal{A})))$ (with $\sigma(\mathcal{A})$ as the set of eigenvalues of $\mathcal{A}$ ) since this is obviously implied by $\mathcal{A}^{T} \mathcal{P}+\mathcal{P} \mathcal{A}+\lambda \mathcal{P}<0$.

Finally, we remark that (18) can be fairly conservative, especially when the system has slow or lightly damped modes that are weakly connected to the output $z_{j}(t)$. Also, the peakto-peak gain may be a poor estimate of the overshoot of the step response.

\section{G. Regional Pole Constraints}

Pole assignment in convex regions of the left-half plane can also be expressed as LMI constraints on the Lyapunov matrix $\mathcal{P}$. To this end, a useful tool is the notion of the LMI region introduced in [11]. An LMI region is any region $\mathcal{R}$ of the complex plane that can be defined as

$$
\mathcal{R}=\left\{z \in C: L+z M+\bar{z} M^{T}<0\right\}
$$

where $L=L^{T}$ and $M$ are fixed real matrices. Note that $\mathcal{R}$ is a convex region in the complex plane. Special cases include vertical strips, disks, horizontal strips, conic sectors, ellipsoids, parabolas, and arbitrary intersections thereof (see [11] for details).

The standard Lyapunov theorem for the open left-half plane $\{z: z+\bar{z}<0\}$ can be generalized to arbitrary LMI regions. Specifically, a matrix $\mathcal{A}$ has all its eigenvalues in the LMI region $\mathcal{R}$ if and only if some LMI involving $\mathcal{A}$ is solvable. This result makes LMI regions particularly appealing for synthesis purposes.

Theorem 2 [11]: The matrix $\mathcal{A}$ has all its eigenvalues in the LMI region $\left\{z \in C:\left[l_{i j}+m_{i j} z+m_{j i} \bar{z}\right]_{i, j}<0\right\}$ if and only if there exists a symmetric $\mathcal{P}$ such that

$$
\left[l_{i j} \mathcal{P}+m_{i j} \mathcal{A}^{T} \mathcal{P}+m_{j i} \mathcal{P} \mathcal{A}\right]_{i, j}<0, \quad \mathcal{P}>0 .
$$

\section{H. Nominal Regulation}

We say that a controller achieves nominal regulation if it is stabilizing and if $\lim _{t \rightarrow \infty} z_{j}(t)=0$ holds for all signals $w_{j}($.$) in the set$

$$
\left\{w_{j}(\cdot) \mid \dot{w}_{j}(t)=S w_{j}(t)\right\} .
$$

This can be interpreted as asymptotically rejecting the disturbance $w=R_{j} w_{j}$ from $z_{j}=L_{j} z$ or, equivalently, letting $C_{j} x+E_{j} u$ track $-D_{j} w_{j}$ asymptotically. The matrix $S$ is called the signal generator.

We want to apply the results of [14] that have been formulated under the following hypotheses:

$$
\begin{aligned}
& \left(\begin{array}{lll}
C_{j} & D_{j} & E_{j}
\end{array}\right)=\left(\begin{array}{lll}
C & F_{j} & 0
\end{array}\right), \quad \sigma(S) \subset \mathbf{C}^{0} \cup \mathbf{C}^{+} \\
& \left(\begin{array}{cc}
A & B_{j} \\
0 & S \\
\hline C & F_{j}
\end{array}\right) \text { is detectable. }
\end{aligned}
$$

The first condition states that the controlled output signal $z_{j}$ for this performance specification is identical to the measured signal $y$. Since any regulating controller is stabilizing, we can dispense with decaying signals which motivate the second property. If the third hypothesis does not hold, one can in fact reduce the signal generator to arrive at this property without causing loss of generality. Now we are ready to provide the solution to the nominal regulation problem as it can be extracted from [14].

Theorem 3: There exists a controller which achieves nominal regulation iff the linear equation

$$
\left(\begin{array}{ll}
A & B \\
C & 0
\end{array}\right)\left(\begin{array}{l}
U \\
V
\end{array}\right)-\left(\begin{array}{c}
\boldsymbol{U} \\
0
\end{array}\right) S=\left(\begin{array}{l}
B_{j} \\
F_{j}
\end{array}\right)
$$


has solutions $\boldsymbol{U}, \boldsymbol{V}$. A controller achieves regulation iff it has a realization

$$
u=\left[\begin{array}{cc|c}
S & C_{K 2} & D_{K 2} \\
0 & A_{K} & B_{K} \\
\hline \boldsymbol{V} & C_{K 1} & D_{K 1}
\end{array}\right] y
$$

where $\boldsymbol{V}$ satisfies (24) (for some $U$ ) and $A_{K}, B_{K}, C_{K 1}, C_{K 2}$, $D_{K 1}$, and $D_{K 2}$ are such that the system

$$
\left(\begin{array}{l}
v_{1} \\
v_{2}
\end{array}\right)=\left[\begin{array}{l|l}
A_{K} & B_{K} \\
\hline C_{K 1} & D_{K 1} \\
C_{K 2} & D_{K 2}
\end{array}\right] y
$$

stabilizes the extended plant

$$
\left(\begin{array}{l}
z \\
y
\end{array}\right)=\left[\begin{array}{cc|ccc}
A & B V & B_{w} & B & 0 \\
0 & S & 0 & 0 & I \\
\hline C_{z} & D_{z} V & D_{z w} & D_{z} & 0 \\
C & 0 & D_{w} & 0 & 0
\end{array}\right]\left(\begin{array}{l}
w \\
v_{1} \\
v_{2}
\end{array}\right) .
$$

Remark 4: Note that (25) can be factorized as

$$
u=\left[\begin{array}{l|ll}
S & 0 & I \\
\hline V & I & 0
\end{array}\right]\left(\begin{array}{l}
v_{1} \\
v_{2}
\end{array}\right), \quad\left(\begin{array}{l}
v_{1} \\
v_{2}
\end{array}\right)=\left[\begin{array}{l|l}
A_{K} & B_{K} \\
\hline C_{K 1} & D_{K 1} \\
C_{K 2} & D_{K 2}
\end{array}\right] y
$$

and that (27) is nothing but the original system precompensated by

$$
u=\left[\begin{array}{l|ll}
S & 0 & I \\
\hline V & I & 0
\end{array}\right]\left(\begin{array}{l}
v_{1} \\
v_{2}
\end{array}\right) .
$$

\section{Robust Regulation}

In many problems it is important to keep up the regulation requirement in the face of uncertainties affecting the plant. The controller achieves robust regulation if it achieves nominal regulation (as defined in the last section), even after slightly perturbing the matrices describing the original system [12]. Classical results about robust regulation apply if

$$
\begin{aligned}
& \left(\begin{array}{lll}
C_{j} & D_{j} & E_{j}
\end{array}\right)=L\left(\begin{array}{lll}
C & F_{j} & 0
\end{array}\right), \quad L \text { of full row rank } \\
& \sigma(S) \subset \mathbf{C}^{0} \cup \mathbf{C}^{+} .
\end{aligned}
$$

The first condition implies that the controlled output signal $z_{j}$ is a linear function $L y$ of the measured output, and the second one can be made since decaying signals are automatically regulated if the system is stabilized.

By the internal model principle, any robust regulator must contain a replica of the signal generator dynamics that can be constructed as follows. Let $l$ be the number of rows of $L$, let $\alpha_{j}+i \beta_{j}, j=1, \cdots, s$, be the list of all pairwise different eigenvalues of $S$ with nonnegative imaginary part, and denote by $s_{j}$ the size of the largest Jordan block corresponding to $\alpha_{j}+i \beta_{j}$. Then define the internal model $(\tilde{S}, \tilde{R})$ as follows.

- If $\beta_{j}>0$, set $U_{j}:=\left(\begin{array}{cc}\alpha_{j} I_{l}, & -\beta_{j} I_{l} \\ \beta_{j} I_{l}, & \alpha_{j} I_{l}\end{array}\right), \quad V_{j}=\left(\begin{array}{cc}I_{l}, & 0 \\ 0, & I_{l}\end{array}\right)$, $W_{j}=\left(\begin{array}{c}0 \\ I_{l}\end{array}\right)$.

- If $\beta_{j}=0$, set $U_{j}:=\alpha_{j} I_{l}, \quad V_{j}=I_{l}, W_{j}=I_{l}$.
Then, define

$$
\begin{aligned}
\tilde{S}_{j}: & =\left(\begin{array}{ccccc}
U_{j} & V_{j} & 0 & \cdots & 0 \\
0 & U_{j} & V_{j} & \cdots & 0 \\
\vdots & & \ddots & \ddots & \vdots \\
0 & \cdots & 0 & U_{j} & V_{j} \\
0 & \cdots & 0 & 0 & U_{j}
\end{array}\right) \\
\tilde{R}_{j} & =\left(\begin{array}{c}
0 \\
0 \\
\vdots \\
0 \\
W_{j}
\end{array}\right), \quad \tilde{S}=\operatorname{diag}\left(S_{1}, \cdots, S_{s}\right) \\
\tilde{R} & =\left(\begin{array}{c}
\tilde{R}_{1} \\
\vdots \\
\tilde{R}_{s}
\end{array}\right)
\end{aligned}
$$

where $\tilde{S}_{j}$ is square and $\tilde{S}_{j}, \tilde{R}_{j}$ have $s_{j}$ block rows.

Then we arrive at the following classical result [12].

Theorem 5: There exists a controller which achieves robust regulation iff

$$
\begin{aligned}
& \left(\begin{array}{cc}
A-\left(\alpha_{j}+i \beta_{j}\right) I & B \\
L C & 0
\end{array}\right) \text { has full row rank } \\
& \text { for all } j=1, \cdots, s .
\end{aligned}
$$

A controller achieves robust regulation iff it has a realization

$$
u=\left[\begin{array}{cc|c}
A_{K} & B_{K 1} & B_{K 2} \\
0 & \tilde{S} & \tilde{R} L \\
\hline C_{K} & D_{K 1} & D_{K 2}
\end{array}\right] y
$$

where $A_{K}, B_{K 1}, B_{K 2}, C_{K}, D_{K 1}$, and $D_{K 2}$ are such that the system

$$
u=\left[\begin{array}{l|ll}
A_{K} & B_{K 1} & B_{K 2} \\
\hline C_{K} & D_{K 1} & D_{K 2}
\end{array}\right]\left(\begin{array}{l}
v \\
y
\end{array}\right)
$$

stabilizes the extended plant

$$
\left(\begin{array}{c}
z \\
v \\
y
\end{array}\right)=\left[\begin{array}{cc|cc}
A & 0 & B_{w} & B \\
\tilde{R} L C & \tilde{S} & \tilde{R} L D_{w} & 0 \\
\hline C_{z} & 0 & D_{z w} & D_{z} \\
0 & I & 0 & 0 \\
C & 0 & D_{w} & 0
\end{array}\right]\left(\begin{array}{l}
w \\
u
\end{array}\right) .
$$

Remark 6: Now, regulator (29) can be factorized as

$$
\begin{aligned}
& u=\left[\begin{array}{l|ll}
A_{K} & B_{K 1} & B_{K 2} \\
\hline C_{K} & D_{K 1} & D_{K 2}
\end{array}\right]\left(\begin{array}{l}
v \\
y
\end{array}\right) \\
& v=\left[\begin{array}{c|c}
\tilde{S} & \tilde{R} L \\
\hline I & 0
\end{array}\right] y
\end{aligned}
$$

and (31) is nothing but the original system postcompensated by

$$
v=\left[\begin{array}{c|c}
\tilde{S} & \tilde{R} L \\
\hline I & 0
\end{array}\right] y .
$$

In contrast to the nominal regulation problem, the internal model is hence put at the output of the system to arrive at the extended system (31). 
In [12] this result is stated with the following modification: replace $\tilde{R}$ by an (varying) $R$ such that $(\widetilde{S}, R)$ is controllable. Hence, as an additional ingredient, we claim that one can fix $R$ to $\tilde{R}$ without loss of generality. Indeed, it is not difficult to show the following algebraic fact: for any $R$ such that $(\tilde{S}, R)$ is controllable, there exists a nonsingular $T$ with $T^{-1} \tilde{S} T=\tilde{S}$ and $T^{-1} R=\tilde{R}$. Hence, if $R$ differs from $\tilde{R}$, we just need to perform a state coordinate change in the controller to transform $R$ back to $\tilde{R}$.

\section{LMI APPROACH TO MULTIOBJECTIVE SYNTHESIS}

In the previous section, several time- and frequency-domain specifications have been expressed as LMI constraints on the closed-loop state-space matrices and Lyapunov functions. These analyses' results are now used for multiobjective synthesis purposes. We begin by formalizing the underlying principle and discussing its merits and limitations. We then propose a systematic procedure to turn analysis results into LMI constraints on the synthesis variables. This procedure relies on a simple change of controller variables to map all LMI's of Section III into a set of affine constraints on the new controller variables and the closed-loop Lyapunov function.

\section{A. Lyapunov Shaping Paradigm}

Our goal is to compute a single LTI controller $K$ that: 1) internally stabilizes the closed-loop and 2) meets certain specifications on a particular set of channels. The specifications under consideration are those listed in Section III. For each specification, we have an analysis result of the form:

$T_{j}$ satisfies the specification $\mathcal{S}_{j}$ if there exists a Lyapunov matrix $\mathcal{P}_{j}>0$ that satisfies some given LMI in $\mathcal{P}_{j}$.

Suppose that our synthesis problem involves $N$ specifications $\mathcal{S}_{1}, \cdots, \mathcal{S}_{N}$ (without restriction on which channel they apply). When gathering the LMI formulation of each specification $\mathcal{S}_{j}$, we end up with a set of matrix inequalities whose variables are:

- the controller matrices $A_{K}, B_{K}, C_{K}, D_{K}$;

- the $N$ Lyapunov matrices $\mathcal{P}_{1}, \cdots, \mathcal{P}_{N}$ (one per specification);

- additional auxiliary variables such as $Q$ ( $H_{2}$ norm), $\lambda, \mu$ (peak-to-peak norm), and $V$ (nominal regulation).

Since expressions like $\mathcal{A}^{T} \mathcal{P}_{j}+\mathcal{P}_{j} \mathcal{A}$ involve products of $\mathcal{P}_{j}$ and the controller variables, the resulting feasibility problem is nonlinear. Hence, it cannot be handled by LMI optimization and does not seem easily tractable numerically.

To recover convexity, we must require that all specifications are enforced by a single closed-loop Lyapunov function $V\left(x_{c l}\right)=x_{c l}^{T} \mathcal{P} x_{c l}$ with $\mathcal{P}>0$. This amounts to imposing the constraint

$$
\mathcal{P}_{1}=\cdots=\mathcal{P}_{N}=\mathcal{P}
$$

This restriction has been extensively used in the state-feedback case [6], [9] and in previous work on mixed $\mathrm{H}_{2} / \mathrm{H}_{\infty}$ synthesis [5], [22]. With (32) in force, all inequalities can be further reduced to LMI's without additional conservatism as shown below.

Before moving to the LMI solution of this simplified problem, we make a few comments on the implications of (32). Clearly, this restriction is stringent and brings conservatism into the design. Nevertheless, the resulting synthesis technique has valuable merits over existing alternatives. First, it is numerically tractable since it leads to an LMI problem. Second, it produces controllers of reasonable order. Finally, it exploits all degrees of freedom in $\mathcal{P}$. Specifically, the Lyapunov matrix $\mathcal{P}$ is shaped by LMI optimization until either all specifications are met or all degrees of freedom are exhausted.

This Lyapunov shaping paradigm offers greater flexibility than standard "optimal" design techniques. For instance, suppose that we need to minimize the $\mathrm{H}_{2}$ norm on one channel, subject to some moderate $H_{\infty}$ performance requirement on another channel. While there may be a large set of Lyapunov matrices $\mathcal{P}$ compatible with the specified $H_{\infty}$ performance, $H_{\infty}$-synthesis techniques based on Riccati equations are unable to exploit these additional degrees of freedom and may return a solution with poor $H_{2}$ performance or unacceptably fast controller dynamics. In contrast, multiobjective LMI synthesis will use these degrees of freedom to optimize the $\mathrm{H}_{2}$ performance or pole location. Thus our approach, while conservative, is nonetheless an improvement over classical synthesis techniques and a valuable tool to fine-tune complex designs.

\section{B. Linearizing Change of Variable}

In the state-feedback case, the simplification (32) makes all inequalities affine in $\mathcal{P}^{-1}$ and $F \mathcal{P}^{-1}$, where $F$ is the statefeedback gain to be determined. It then only takes the change of variables $\boldsymbol{X}=\mathcal{P}^{-1}, \boldsymbol{F}=F \mathcal{P}^{-1}$ to turn all constraints into LMI's. A similar approach was long believed beyond reach in the output-feedback case. However, recent results [11], [24], [30] have proved otherwise. This critical change of variables is defined next. Note that we use boldface letters to emphasize the LMI optimization variables.

Let $n$ be the number of states of the plant (size of $A$ ), and let $k$ be the order of the controller. Partition $\mathcal{P}$ and $\mathcal{P}^{-1}$ as

$$
\mathcal{P}=\left(\begin{array}{cc}
Y & N \\
N^{T} & \star
\end{array}\right), \quad \mathcal{P}^{-1}=\left(\begin{array}{cc}
X & M \\
M^{T} & \star
\end{array}\right)
$$

where $\boldsymbol{X}$ and $\boldsymbol{Y}$ are $n \times n$ and symmetric. From $\mathcal{P P}^{-1}=I$, we infer $\mathcal{P}\left(\begin{array}{l}\boldsymbol{X} \\ M^{T}\end{array}\right)=\left(\begin{array}{l}I \\ 0\end{array}\right)$ which leads to

$$
\begin{aligned}
\mathcal{P} \Pi_{1} & =\Pi_{2} \text { with } \Pi_{1}:=\left(\begin{array}{cc}
\boldsymbol{X} & I \\
M^{T} & 0
\end{array}\right) \\
\Pi_{2} & :=\left(\begin{array}{cc}
I & \boldsymbol{Y} \\
0 & N^{T}
\end{array}\right) .
\end{aligned}
$$

Let us now define the change of controller variables as follows:

$$
\left\{\begin{aligned}
\hat{\boldsymbol{A}}:= & N A_{K} M^{T}+N B_{K} C \boldsymbol{X}+\boldsymbol{Y} B C_{K} M^{T} \\
& +\boldsymbol{Y}\left(A+B D_{K} C\right) \boldsymbol{X} \\
\hat{\boldsymbol{B}}:= & N B_{K}+\boldsymbol{Y} B D_{K} \\
\hat{\mathbf{C}}:= & C_{K} M^{T}+D_{K} C \boldsymbol{X} \\
\hat{\boldsymbol{D}}:= & D_{K} .
\end{aligned}\right.
$$


Note that the new variables $\hat{\boldsymbol{A}}, \hat{\boldsymbol{B}}, \hat{\mathbf{C}}$ have dimensions $n \times n$, $n \times n_{u}$, and $n_{y} \times n$, respectively. If $M$ and $N$ have full row rank, and if $\hat{A}, \hat{B}, \hat{\mathbf{C}}, \hat{D}, X$, and $Y$ are given, we can always compute controller matrices $A_{K}, B_{K}, C_{K}, D_{K}$ satisfying (35). If $M$ and $N$ are square $(k=n)$ and invertible matrices, then $A_{K}, B_{K}, C_{K}$, and $D_{K}$ are unique. For fullorder design, one can always assume that $M$ and $N$ have full row rank. Hence the variables $A_{K}, B_{K}, C_{K}, D_{K}$ can be replaced by $\hat{A}, \hat{B}, \hat{\mathbf{C}}, \hat{D}$ without loss of generality. Note that this same change of variables was already used in [17] in the context of pure $H_{\infty}$ control and in [11] and [30] for special cases of the general multiobjective problem discussed in this paper.

The motivation for this transformation lies in the following identities derived from (34) and (35) after a short calculation:

$$
\begin{aligned}
& \Pi_{1}^{T} \mathcal{P} \mathcal{A} \Pi_{1}=\Pi_{2}^{T} \mathcal{A} \Pi_{1}=\left(\begin{array}{cc}
A \boldsymbol{X}+B \hat{\mathbf{C}} & A+B \hat{D} C \\
\hat{A} & \boldsymbol{Y} A+\hat{B} C
\end{array}\right) \\
& \Pi_{1}^{T} \mathcal{P} \mathcal{B}_{j}=\Pi_{2}^{T} \mathcal{B}_{j}=\left(\begin{array}{l}
B_{j}+B \hat{D} F_{j} \\
\boldsymbol{Y} B_{j}+\hat{\boldsymbol{B}} F_{j}
\end{array}\right) \\
& \mathcal{C}_{j} \Pi_{1}=\left(C_{j} \boldsymbol{X}+E_{j} \hat{\mathbf{C}} C_{j}+E_{j} \hat{D} C\right) \\
& \Pi_{1}^{T} \mathcal{P} \Pi_{1}=\Pi_{1}^{T} \Pi_{2}=\left(\begin{array}{cc}
\boldsymbol{X} & I \\
I & \boldsymbol{Y}
\end{array}\right) .
\end{aligned}
$$

In light of these identities, we are ready to show how synthesis LMI's can be derived from the analysis results of Section III via a suitable congruence transformation. A detailed proof is given only for the generalized $H_{2}$ problem.

Fix $\alpha>0$ and suppose that (16) holds for some $\mathcal{P}>0$ and some controller with realization $\left(A_{K}, B_{K}, C_{K}, D_{K}\right)$. We can assume without loss of generality that this controller is of order at least $n$ and that $M$ and $N$ in (33) have full row rank (see [17] for details). By (34), and since $\mathcal{P}$ is nonsingular, $\Pi_{1}$ has full column rank. If we perform a congruence transformation with diag $\left(\Pi_{1}, I\right)$ on both inequalities (16), we obtain

$$
\begin{aligned}
\left(\begin{array}{cc}
\Pi_{1}^{T} \mathcal{A}^{T} \mathcal{P} \Pi_{1}+\Pi_{1}^{T} \mathcal{P} \mathcal{A} \Pi_{1} & \Pi_{1}^{T} \mathcal{P} \mathcal{B}_{j} \\
\mathcal{B}_{j}^{T} \mathcal{P} \Pi_{1} & -I
\end{array}\right)<0 \\
\left(\begin{array}{cc}
\Pi_{1}^{T} \mathcal{P} \Pi_{1} & \Pi_{1}^{T} \mathcal{C}_{j}^{T} \\
\mathcal{C}_{j} \Pi_{1} & \alpha I
\end{array}\right)>0 .
\end{aligned}
$$

Now we just need to replace $\Pi_{1}^{T} \mathcal{P} \mathcal{A} \Pi_{1}, \Pi_{1}^{T} \mathcal{P} \mathcal{B}_{j}, \mathcal{C}_{j} \Pi_{1}$, and $\Pi_{1}^{T} \mathcal{P} \Pi_{1}$ by their explicit expressions (36) to arrive at (38), shown at the bottom of the page. These inequalities/equations are clearly affine in $\hat{A}, \hat{B}, \hat{\mathbf{C}}, \hat{D}, \boldsymbol{X}, Y$. Thus we have proved that the solvability of these LMI's is necessary for the existence of a stabilizing controller rendering $\|T\|_{g}^{2}<\alpha$.

Let us now reverse the argument and assume that we have found solutions to the LMI's (38). First we need to construct
$M, N$, and $\mathcal{P}$ that satisfy (34). Looking at the left upper block of $\mathcal{P} \Pi_{1}=\Pi_{2}, M$ and $N$ should be chosen such that

$$
M N^{T}=I-X Y .
$$

By $\left(\begin{array}{c}\boldsymbol{X}, I \\ I, Y\end{array}\right)>0$, we infer $\boldsymbol{Y}>0, \boldsymbol{X}-\boldsymbol{Y}^{-1}>0$ such that $I-X Y$ is nonsingular. Hence, one can always find square and nonsingular $M$ and $N$ satisfying (39). After defining $\Pi_{1}$, $\Pi_{2}$ as in (34), we observe that these matrices are nonsingular, and we can set $\mathcal{P}:=\Pi_{2} \Pi_{1}^{-1}$ to obtain (34). Since $M$ and $N$ are nonsingular, (35) can be solved for $D_{K}, C_{K}, B_{K}$, and $A_{K}$ in this order. Since (34) and (35) imply (36), we know that (37) and (38) are identical. Recalling that $\Pi_{1}$ is square and nonsingular, we can reverse the congruence transformation with diag $\left(\Pi_{1}, I\right)$ to obtain (16) from (37). Hence the constructed controller indeed leads to $\left\|T_{j}\right\|_{g}^{2}<\alpha$.

Let us finally observe that the synthesis LMI's are also affine in $\alpha$. Hence, minimizing $\alpha$ subject to the LMI constraints (38) is also an LMI problem. For the controller computation, however, one should keep in mind that $I-X Y$ ought to be well conditioned to avoid ill-conditioned inversions of the matrices $M$ and $N$. Unfortunately, $I-X Y$ will be nearly singular if the constraint (41) is saturated at the optimum. To avoid such difficulties, we advise the following remedy: choose some nearly optimal value of $\alpha$, and include the LMI

$$
\left(\begin{array}{ll}
X & t I \\
t I & Y
\end{array}\right)>0
$$

with the additional variable $t$ and maximize $t$. This procedure maximizes the minimal eigenvalue of $\boldsymbol{X Y}$ and, hence, pushes it away from one such that $I-X Y$ is expected to be well conditioned.

\section{Synthesis LMI's and Controller Computation}

The discussion of the last section allows us to extract a recipe for getting to an LMI synthesis result on the basis of an LMI analysis result: suppose the analysis result is formulated in terms of LMI's in the blocks $\mathcal{P}, \mathcal{P} \mathcal{A}, \mathcal{P} \mathcal{B}_{j}, \mathcal{C}_{j}$, $\mathcal{D}_{j}$, and their transposes, and suppose one can find congruence transformations of these LMI's that involve the block $\Pi_{1}$ and that transform the original LMI's into LMI's in the blocks $\Pi_{1}^{T} \mathcal{P} \Pi_{1}, \Pi_{1}^{T} \mathcal{P} \mathcal{A} \Pi_{1}, \Pi_{1}^{T} \mathcal{P} \mathcal{B}_{j}, \mathcal{C}_{j} \Pi_{1}, \mathcal{D}_{j}$ and their transposes. If one substitutes all the appearing blocks by the formulas (36), one arrives at the corresponding synthesis LMI's in $\hat{A}, \hat{B}, \hat{\mathbf{C}}, \hat{D}, X, Y$ and possible auxiliary variables. After solving the synthesis LMI's, the controller construction proceeds as follows: find nonsingular matrices $M, N$ to satisfy

$$
\begin{aligned}
\left(\begin{array}{ccc}
A \boldsymbol{X}+\boldsymbol{X} A^{T}+B \hat{\mathbf{C}}+(B \hat{\mathbf{C}})^{T} & \hat{\boldsymbol{A}}^{T}+(A+B \hat{\boldsymbol{D}} C) & B_{j}+B \hat{\boldsymbol{D}} F_{j} \\
\hat{\boldsymbol{A}}+(A+B \hat{\boldsymbol{D}} C)^{T} & A^{T} \boldsymbol{Y}+\boldsymbol{Y} A+\hat{\boldsymbol{B}} C+(\hat{\boldsymbol{B} C})^{T} & \boldsymbol{Y} B_{j}+\hat{\boldsymbol{B}} F_{j} \\
\left(B_{j}+B \hat{\boldsymbol{D}} F_{j}\right)^{T} & \left(\boldsymbol{Y} B_{j}+\hat{\boldsymbol{B}} F_{j}\right)^{T} & -I
\end{array}\right)<0 & \\
\left(\begin{array}{ccc}
\boldsymbol{X} & I & \left(C_{j} \boldsymbol{X}+E_{j} \hat{\mathbf{C}}\right)^{T} \\
I & \boldsymbol{Y} & \left(C_{j}+E_{j} \hat{\boldsymbol{D}} C\right)^{T} \\
C_{j} \boldsymbol{X}+E_{j} \hat{\mathbf{C}} & C_{j}+E_{j} \hat{D} C & \alpha I
\end{array}\right)>0, & D_{j}+E_{j} \hat{\boldsymbol{D}} F_{j}=0
\end{aligned}
$$


$M N^{T}=I-X Y$ and define the controller by

$$
\left\{\begin{aligned}
& D_{K}:=\hat{\boldsymbol{D}} \\
& C_{K}:=\left(\hat{\mathbf{C}}-D_{K} C \boldsymbol{X}\right) M^{-T} \\
& B_{K}:= N^{-1}\left(\hat{\boldsymbol{B}}-Y B D_{K}\right) \\
& A_{K}:= N^{-1}\left(\hat{A}-N B_{K} C \boldsymbol{X}-\boldsymbol{Y} B C_{K} M^{T}\right. \\
&\left.\quad-\boldsymbol{Y}\left(A+B D_{K} C\right) \boldsymbol{X}\right) M^{-T} .
\end{aligned}\right.
$$

This gives a formal description of all problems to which we can apply the controller parameter transformation in order to obtain the synthesis LMI's. The proof is literally the same as for the generalized $H_{2}$ problem discussed in Section IVB. Note that the necessity part of the proof does not restrict the order of the controller and that the construction in the sufficiency part leads to a controller that is of the same order as the plant. Finally, we stress that this procedure does not introduce any conservatism: if the analysis result does not involve conservatism, the synthesis result does neither. If combining several of these specification into a multiobjective design, additional conservatism is only introduced through using a common Lyapunov function, at the benefit of being able to restrict the order of the controller to that of the plant.

For nominal and robust regulation, we need a slight modification of this procedure that is discussed in Section V-G.

\section{A CATALOG OF LMI'S FOR FULL-ORDER SyNTHESIS}

For completeness, this section lists the synthesis LMI's attached to each specification or objective considered in Section III. These synthesis LMI's are readily derived from the analysis results of Section III by applying the systematic procedure described in Section IV-B. No new proof is required to justify these results, the proof in IV-B being generic. This section is meant as a catalog where one can easily find the appropriate LMI formulation for each particular specification. For instance, suppose that the design problem involves an $H_{\infty}$ constraint on channel one, an $\mathrm{H}_{2}$ constraint on channel two, and a regional pole placement constraint. One would then find the corresponding synthesis LMI's on the list below, gather them in a single system of LMI's, and solve this LMI system numerically to derive a solution of the multiobjective problem under consideration.
Since the requirement $\mathcal{P}>0$ is common to all analysis results of Section III, the constraint

$$
\left(\begin{array}{cc}
X & I \\
I & Y
\end{array}\right)>0
$$

should always be included in the list of synthesis LMI's, either explicitly or as part of some other LMI constraint [see, e.g., the second LMI in (38)].

\section{A. $H_{\infty}$ Synthesis}

The analysis LMI's (8) are transformed with $\Pi_{1}$ and $\operatorname{diag}\left(\Pi_{1}, I, I\right)$. Then, (36) leads to the synthesis LMI as in (42), shown at the bottom of the page, where $*$ replaces blocks that are readily inferred by symmetry. Since $\gamma$ enters linearly, it can be directly minimized by LMI optimization to find the smallest achievable $H_{\infty}$ norm. As a single objective problem, no conservatism is involved. Imposing independent $H_{\infty}$ constraints on several different channels just amounts to incorporating (42) for each individual channel and introduces conservatism.

\section{B. General Quadratic Constraints}

With the congruence transformations $\Pi_{1}$ and $\operatorname{diag}\left(\Pi_{1}, I, I\right)$, we obtain the synthesis LMI given in (43), also shown at the bottom of the page, where $(1,1),(1$, $2)$, and $(2,2)$ refer to the corresponding blocks in $(42)$. Instead of fixing $V_{j}$ and $\Sigma_{j}$, we can allow some free parameters in these matrices. Then, it is clearly possible to minimize or maximize a linear function in these free parameters. The synthesis LMI's for designing a strictly passive system are obtained by just setting $Q_{j}=0, \Sigma_{j}=I, V_{j}=0, W_{j}=-I$ (such that the fourth block row and column can be canceled); this extends [31] to general systems. Again, at the expense of conservatism, we can include as many quadratic constraints on different channels as desired.

\section{C. $\mathrm{H}_{2}$ and Generalized $\mathrm{H}_{2}$ Synthesis}

The synthesis LMI's for generalized $H_{2}$ control have been given in (38). With the same congruence transformation $\operatorname{diag}\left(\Pi_{1}, I\right)$, we obtain from the analysis LMI's (14) for the standard $\mathrm{H}_{2}$ problem the synthesis LMI's, as in (v), shown at the bottom of the next page.

$$
\begin{aligned}
& \left(\begin{array}{cccc}
A \boldsymbol{X}+\boldsymbol{X} A^{T}+B \hat{\mathbf{C}}+(B \hat{\mathbf{C}})^{T} & \hat{\boldsymbol{A}}^{T}+(A+B \hat{\boldsymbol{D}} C) & * & * \\
\hat{\boldsymbol{A}}+(A+B \hat{\boldsymbol{D}} C)^{T} & A^{T} \boldsymbol{Y}+\boldsymbol{Y} A+\hat{\boldsymbol{B} C}+(\hat{\boldsymbol{B} C})^{T} & * & * \\
\left(B_{j}+B \hat{\boldsymbol{D}} F_{j}\right)^{T} & \left(\boldsymbol{Y} B_{j}+\hat{\boldsymbol{B}} F_{j}\right)^{T} & -\gamma I & * \\
C_{j} \boldsymbol{X}+E_{j} \hat{\mathbf{C}} & C_{j}+E_{j} \hat{\boldsymbol{D}} C & D_{j}+E_{j} \hat{\boldsymbol{D}} F_{j} & -\gamma I
\end{array}\right)<0 \\
& \left(\begin{array}{cccc}
(1,1) & (1,2) & \left(B_{j}+B \hat{\boldsymbol{D}} F_{j}\right)+\left(C_{j} \boldsymbol{X}+E_{j} \hat{\mathbf{C}}\right)^{T} W_{j} & \left(C_{j} \boldsymbol{X}+E_{j} \hat{\mathbf{C}}\right)^{T} Q_{j} \\
* & (2,2) & \left(\boldsymbol{Y} B_{j}+\hat{B} F_{j}\right)+\left(C_{j}+E_{j} \hat{D} C\right)^{T} W_{j} & \left(C_{j}+E_{j} \hat{D} C\right)^{T} Q_{j} \\
* & * & V_{j}+W_{j}^{T}\left(D_{j}+E_{j} \hat{D} F_{j}\right)+\left(D_{j}+E_{j} \hat{D} F_{j}\right)^{T} W_{j} & \left(D_{j}+E_{j} \hat{\boldsymbol{D}} F_{j}\right)^{T} Q_{j} \\
* & * & * & -\Sigma_{j}
\end{array}\right)<0
\end{aligned}
$$


These involve the auxiliary variables $Q$ and $\nu$ which enter linearly. Hence, $\nu$ can be directly minimized by LMI optimization. Note that synthesis results for a variety of generalized $H_{2}$ criteria can be derived by simply replacing $\operatorname{Tr}(Q)$ with any affine function $f(Q)$ mapping symmetric matrices into symmetric matrices [30].

\section{Synthesis for Bounds on Peak Impulse Response}

The congruence transformations $\Pi_{1}$ and diag $\left(\Pi_{1}, I\right)$ transform (17) into $D_{j}+E_{j} \hat{\boldsymbol{D}} F_{j}=0$ and (w), shown at the bottom of the page. One can directly minimize the upper bound $\xi$ on the peak impulse response. We stress again that even the single objective synthesis result involves conservatism since the analysis result does.

\section{E. Synthesis with Bound on the Peak-to-Peak Gain}

Using the congruence transformations $\operatorname{diag}\left(\Pi_{1}, I\right)$, $\operatorname{diag}\left(\Pi_{1}, I, I\right)$, one arrives at the following synthesis inequalities: $\lambda>0$ and those in (44), shown at the bottom of the page. Note that the auxiliary variable $\lambda$ enters nonlinearly. However, if we fix $\lambda>0$, we can minimize $\zeta$ to obtain an infimal upper bound $\zeta_{*}(\lambda)$ of the peak-to-peak norm that clearly depends on $\lambda$. Hence, to improve this upper bound, we have to further minimize $\zeta_{*}(\lambda)$ over $\lambda>0$, which involves solving a one-dimensional minimization problem.

\section{F. Regional Pole Placement}

The matrix $\operatorname{diag}\left(\Pi_{1}, \cdots, \Pi_{1}\right)$ transforms (22) to the LMI

$$
\begin{gathered}
\left(l_{j k}\left(\begin{array}{cc}
\boldsymbol{X} & I \\
I & \boldsymbol{Y}
\end{array}\right)+m_{j k}\left(\begin{array}{cc}
A \boldsymbol{X}+B \hat{\mathbf{C}} & A+B \hat{\boldsymbol{D}} C \\
\hat{\boldsymbol{A}} & \boldsymbol{Y} A+\hat{\boldsymbol{B} C}
\end{array}\right)^{T}\right. \\
\left.+m_{k j}\left(\begin{array}{cc}
A X+B \hat{\mathbf{C}} & A+B \hat{\boldsymbol{D} C} \\
\hat{\boldsymbol{A}} & \boldsymbol{Y} A+\hat{\boldsymbol{B} C}
\end{array}\right)\right)_{j, k}<0 .
\end{gathered}
$$

We can include this inequality in any of the design problems discussed so far to enforce that the eigenvalues of $\mathcal{A}$ (and hence the poles of the closed-loop system) are contained in the LMI region (21).

\section{G. Nominal Regulation}

Theorem 3 suggests the following procedure to design a controller that achieves regulation and leads to any of the specifications we have listed so far: test whether (24) is solvable. Then, try to achieve the desired specification with the controller (26) for the extended system (27) and some $V$ for which there exists a $U$ such that (24) holds.

The central technical difficulty in this procedure arises from the new unknown $V$ in the plant description (27) which we need to vary in a linear manifold determined by the solution set of (24). If (24) does not have a unique solution, there are free parameters that enter the synthesis inequalities nonlinearly. In general, such problems are hard to resolve. Due to the

$$
\begin{aligned}
& \left(\begin{array}{ccc}
A \boldsymbol{X}+\boldsymbol{X} A^{T}+B \hat{\mathbf{C}}+(B \hat{\mathbf{C}})^{T} & \hat{\boldsymbol{A}}^{T}+(A+B \hat{\boldsymbol{D}} C) & B_{j}+B \hat{\boldsymbol{D}} F_{j} \\
\hat{\boldsymbol{A}}+(A+B \hat{\boldsymbol{D}} C)^{T} & A^{T} \boldsymbol{Y}+\boldsymbol{Y} A+\hat{\boldsymbol{B}} C+(\hat{\boldsymbol{B}} C)^{T} & \boldsymbol{Y} B_{j}+\hat{\boldsymbol{B}} F_{j} \\
\left(B_{j}+B \hat{\boldsymbol{D}} F_{j}\right)^{T} & \left(\boldsymbol{Y} B_{j}+\hat{\boldsymbol{B}} F_{j}\right)^{T} & -I
\end{array}\right)<0 \\
& \left(\begin{array}{ccc}
X & I & \left(C_{j} X+E_{j} \hat{\mathbf{C}}\right)^{T} \\
I & Y & \left(C_{j}+E_{j} \hat{D} C\right)^{T} \\
C_{j} X+E_{j} \hat{\mathbf{C}} & C_{j}+E_{j} \hat{D} C & Q
\end{array}\right)>0 \\
& \operatorname{Tr}(Q)<\nu, \quad D_{j}+E_{j} \hat{D} F_{j}=0 \\
& \left(\begin{array}{cc}
A \boldsymbol{X}+\boldsymbol{X} A^{T}+B \hat{\mathbf{C}}+(B \hat{\mathbf{C}})^{T} & \hat{\boldsymbol{A}}^{T}+(A+B \hat{\boldsymbol{D}} C) \\
\hat{\boldsymbol{A}}+(A+B \hat{\boldsymbol{D}} C)^{T} & A^{T} \boldsymbol{Y}+\boldsymbol{Y} A+\hat{\boldsymbol{B} C}+(\hat{\boldsymbol{B} C})^{T}
\end{array}\right)<0 \\
& \left(\begin{array}{ccc}
X & I & B_{j}+B \hat{\boldsymbol{D}} F_{j} \\
I & \boldsymbol{Y} & \boldsymbol{Y} B_{j}+\hat{\boldsymbol{B}} F_{j} \\
\left(B_{j}+B \hat{\boldsymbol{D}} F_{j}\right)^{T} & \left(\boldsymbol{Y} B_{j}+\hat{\boldsymbol{B}} F_{j}\right)^{T} & \xi I
\end{array}\right)>0 \\
& \left(\begin{array}{ccc}
X & I & \left(C_{j} X+E_{j} \hat{\mathbf{C}}\right)^{T} \\
I & Y & \left(C_{j}+E_{j} \hat{D} C\right)^{T} \\
C_{j} X+E_{j} \hat{\mathbf{C}} & C_{j}+E_{j} \hat{\boldsymbol{D} C} & \xi I
\end{array}\right)>0
\end{aligned}
$$

$$
\begin{aligned}
& \left(\begin{array}{ccc}
A \boldsymbol{X}+\boldsymbol{X} A^{T}+B \hat{\mathbf{C}}+(B \hat{\mathbf{C}})^{T}+\lambda \boldsymbol{X} & { }^{*} & * \\
\hat{\boldsymbol{A}}+(A+B \hat{\boldsymbol{D}} C)^{T}+\lambda I & A^{T} \boldsymbol{Y}+\boldsymbol{Y} A+\hat{\boldsymbol{B} C}+(\hat{\boldsymbol{B} C})^{T}+\lambda \boldsymbol{Y} & * \\
\left(B_{j}+B \hat{\boldsymbol{D}} F_{j}\right)^{T} & \left(\boldsymbol{Y} B_{j}+\hat{\boldsymbol{B}} F_{j}\right)^{T} & -\mu I
\end{array}\right)<0 \\
& \left(\begin{array}{cccc}
\lambda \boldsymbol{X} & \lambda I & 0 & * \\
\lambda I & \lambda \boldsymbol{Y} & 0 & * \\
0 & 0 & (\zeta-\mu) I & * \\
C_{j} \boldsymbol{X}+E_{j} \hat{\mathbf{C}} & C_{j}+E_{j} \hat{D} C & D_{j}+E_{j} \hat{D} F_{j} & \zeta I
\end{array}\right)>0
\end{aligned}
$$


particular structural dependence, however, the present problem is fully tractable. Indeed, one has to work with a suitable modification of $\Pi_{1}$ to arrive at tractable LMI's. For this purpose we define

$$
W_{U}:=\left(\begin{array}{cc}
I & \boldsymbol{U} \\
0 & I
\end{array}\right)
$$

Then, one easily obtains

$$
W_{U}^{-1}\left(\begin{array}{cc}
A & B V \\
0 & S
\end{array}\right) W_{U}=\left(\begin{array}{cc}
A & B_{j} \\
0 & S
\end{array}\right)
$$

and

$$
\left(\begin{array}{ll}
C & 0
\end{array}\right) W_{U}=\left(\begin{array}{ll}
C & F_{j}
\end{array}\right)
$$

from (24). Let us modify $\Pi_{1}$ to

$$
\Pi_{U}=\Pi_{1}\left(\begin{array}{cc}
I & 0 \\
0 & W_{U}
\end{array}\right)=\left(\begin{array}{cc}
\boldsymbol{X} & W_{U} \\
M^{T} & 0
\end{array}\right)
$$

and consider the blocks (36) for the extended system (27) and with $\Pi_{1}$ replaced by $\Pi_{U}$. The block $\Pi_{U}^{T} \mathcal{P} \mathcal{A} \Pi_{U}$ is given by (x), shown at the bottom of the page. These formulas motivate the introduction of the new variables

$$
\begin{aligned}
& \tilde{\boldsymbol{Y}}:=W_{U}^{T} \boldsymbol{Y} W_{U}, \quad \tilde{\boldsymbol{A}}:=W_{U}^{T} \hat{\boldsymbol{A}} \\
& \tilde{\boldsymbol{B}}:=W_{U}^{T} \hat{\boldsymbol{B}}, \quad \tilde{\mathbf{C}}:=\left(\begin{array}{cc}
0 & \boldsymbol{V} \\
0 & 0
\end{array}\right) \boldsymbol{X}+\hat{\mathbf{C}}
\end{aligned}
$$

and due to the nonsingularity of $W_{U}$, this is just another parameter transformation. If we exploit (46), we obtain (y), as shown at the bottom of the page. Obviously, all these blocks depend affinely on the variables $U, V, X, \tilde{Y}, \tilde{A}, \tilde{B}, \tilde{\mathbf{C}}, \hat{D}$.

We arrive at the following procedure for performing multiobjective synthesis including nominal regulation: in any analysis LMI, substitute $\mathcal{P}, \mathcal{P} \mathcal{A}, \mathcal{P} \mathcal{B}_{j}, \mathcal{C}_{j}$ by these new formulas [instead of those in (36)] and add the linear constraint (24) for the extra variables $\boldsymbol{U}, \boldsymbol{V}$. Test the resulting synthesis LMI's for solvability. With a solution, the controller is constructed as follows: reverse (47) as

$$
\begin{aligned}
& \boldsymbol{Y}=W_{U}^{-T} \tilde{\boldsymbol{Y}} W_{U}^{-1}, \quad \hat{\boldsymbol{A}}=W_{U}^{-T} \tilde{\boldsymbol{A}} \\
& \hat{\boldsymbol{B}}=W_{U}^{-T} \tilde{\boldsymbol{B}}, \quad \hat{\mathbf{C}}=\tilde{\mathbf{C}}-\left(\begin{array}{cc}
0 & \boldsymbol{V} \\
0 & 0
\end{array}\right) \boldsymbol{X}
\end{aligned}
$$

to obtain $\boldsymbol{U}, \boldsymbol{V}, \boldsymbol{X}, \boldsymbol{Y}, \hat{\boldsymbol{A}}, \hat{\boldsymbol{B}}, \hat{\mathbf{C}}, \hat{\boldsymbol{D}}$; find square $M, N$ with $M N^{T}=I-X Y$, and define the parameters $A_{K}, B_{K}, C_{K}$, and $D_{K}$ as in (40) for the extended system (27); partition the rows of $C_{K}$ and $D_{K}$ as in (26); then, the desired controller is given as (25) and its order is bounded by the order of the plant (size of $A_{K}$ ) plus the size of $S$.

Note that this procedure does not introduce conservatism. For a single-objective specification with an exact LMI analysis/synthesis characterization, it leads to an exact solution of the corresponding problem with nominal regulation, and in the other cases, conservatism is only introduced through nonexact analysis tests or through the use of a common Lyapunov function for multiple objectives.

For nominal regulation, the most related results are found in [10], where a special $H_{\infty}$ problem (for coprime factor uncertainty) is discussed under the hypothesis that (24) has a unique solution. Incorporating a general nominal regulation requirement [without hypothesis on (24)] in all the other single-objective or mixed problems are new design goals that found a complete solution.

\section{H. Robust Regulation}

Contrary to nominal regulation, the internal model and the extended system in Theorem 5 are fixed such that they pose no problem for including the requirement of robust regulation in any of the specifications listed so far without introducing extra conservatism. Build (31), solve the corresponding synthesis problems for this extended plant with a controller (30), and define the final controller by (29). Its order is bounded by that of the plant plus the size of $\tilde{S}$.

One can in fact prove that the controller (29) achieves regulation for any LTI system (1) for which (30) stabilizes (31). Suppose that (1) is affected by perturbations $w=$

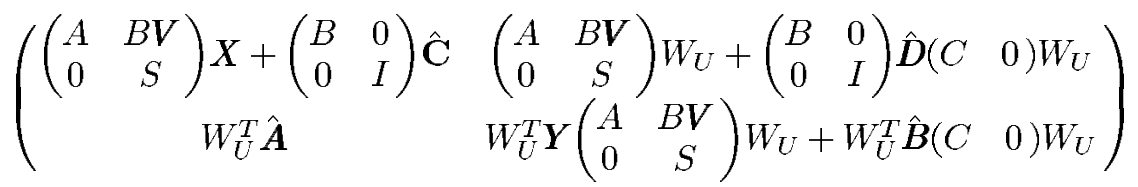

$$
\begin{aligned}
& \Pi_{U}^{T} \mathcal{P} \mathcal{A} \Pi_{U}=\left(\begin{array}{cc}
\left(\begin{array}{cc}
A & 0 \\
0 & S
\end{array}\right) \boldsymbol{X}+\left(\begin{array}{cc}
B & 0 \\
0 & I
\end{array}\right) \tilde{\mathbf{C}} & \left(\begin{array}{cc}
A & A \boldsymbol{U}+B V \\
0 & S
\end{array}\right)+\left(\begin{array}{cc}
B & 0 \\
0 & I
\end{array}\right) \hat{\boldsymbol{D}}\left(\begin{array}{ll}
C & F_{j}
\end{array}\right) \\
\tilde{\boldsymbol{A}} & \tilde{\boldsymbol{Y}}\left(\begin{array}{cc}
A & B_{j} \\
0 & S
\end{array}\right)+\tilde{\boldsymbol{B}}\left(\begin{array}{ll}
C & F_{j}
\end{array}\right)
\end{array}\right) \\
& \Pi_{U}^{T} \mathcal{P} \Pi_{U}=\left(\begin{array}{cc}
\boldsymbol{X} & \left(\begin{array}{cc}
I & \boldsymbol{U} \\
0 & I
\end{array}\right) \\
\left(\begin{array}{cc}
I & 0 \\
\boldsymbol{U}^{T} & I
\end{array}\right) & \tilde{\boldsymbol{Y}}
\end{array}\right), \quad \Pi_{U}^{T} \mathcal{P} \mathcal{B}_{j}=\left(\begin{array}{c}
\left(\begin{array}{c}
B_{j} \\
0
\end{array}\right)+\left(\begin{array}{cc}
B & 0 \\
0 & I
\end{array}\right) \hat{\boldsymbol{D}} F_{j} \\
\tilde{\boldsymbol{Y}}\left(\begin{array}{c}
B_{j} \\
0
\end{array}\right)+\tilde{\boldsymbol{B}} F_{j},
\end{array}\right) \\
& \left.\mathcal{C}_{j} \Pi_{U}=\left(\begin{array}{ll}
\left(C_{j}\right. & 0
\end{array}\right) \boldsymbol{X}+\left(\begin{array}{llll}
E_{j} & 0
\end{array}\right) \tilde{\mathbf{C}} \quad\left(\begin{array}{lll}
C_{j} & C_{j} \boldsymbol{U}+E_{j} \boldsymbol{V}
\end{array}\right)+\left(\begin{array}{ll}
E_{j} & 0
\end{array}\right) \hat{\boldsymbol{D}}\left(C \quad F_{j}\right)\right)
\end{aligned}
$$


$R_{j} \Delta L_{j} z$ with a (stable) LTI $\Delta$ in some class $\Delta$. Let us assume that one can design a controller (30) which robustly stabilizes (31) for this same class of uncertainties (e.g., by solving an $H_{\infty}$ or passivity problem or any other robust stability specification given in our list). Then, the controller (29) achieves regulation for all perturbed systems (1), simply because (30) stabilizes all the perturbed extended systems (31). Hence, one can guarantee regulation for the (possibly large) class of uncertainties $\Delta$, and this solves the problem of robust regulation in the large.

The present result extends those in [1] and [2] to signal generators that are not necessarily diagonizable and to the variety of mixed problems considered in this paper.

\section{A REMARK ON REDUCED-ORDER CONTROLLERS}

In all mixed problems discussed in this paper, the constructed controllers have the same dimension as the plant (possibly plus internal model in regulation). For completeness and only as a theoretical insight, we include a characterization for the existence of controllers that have dimension $n-k<n$. Let us assume w.l.o.g. that

$$
\left(\begin{array}{c}
B \\
D_{z}
\end{array}\right) \text { and }\left(\begin{array}{c}
C^{T} \\
D_{w}^{T}
\end{array}\right) \text { have full column rank. }
$$

To be specific we outline the result for the generalized $\mathrm{H}_{2}$ problem.

Suppose a controller of dimension $n-k<n$ leads to (16). As earlier, we obtain (34) where the matrices $M, N$ are of size $n \times(n-k)$ and can be assumed to have full column rank. Hence, $\Pi_{1}$ has a kernel of dimension $k$ such that all the blocks $\Pi_{1}^{T} \mathcal{P} \mathcal{A} \Pi_{1}, \Pi_{1}^{T} \mathcal{A}^{T} \mathcal{P} \Pi_{1}, \mathcal{B}_{w}^{T} \mathcal{P} \Pi_{1}, \mathcal{C}_{z} \Pi_{1}$, and $\Pi_{1}^{T} \mathcal{P} \Pi_{1}$ vanish on this kernel. Using (48), it is easily seen that the three blocks

$$
\begin{aligned}
& \left(\begin{array}{cc}
X & I \\
I & Y
\end{array}\right), \quad\left(\begin{array}{cc}
A \boldsymbol{X}+B \hat{\mathbf{C}} & B \hat{\boldsymbol{D}} \\
\hat{A} & \hat{B}
\end{array}\right)^{T}, \\
& \left(\begin{array}{cc}
\hat{\mathbf{C}} & \hat{\boldsymbol{D}} C \\
\hat{\boldsymbol{A}} & \boldsymbol{Y} A+\hat{\boldsymbol{B}} C
\end{array}\right)
\end{aligned}
$$

vanish on the very same subspace. Moreover, since $\operatorname{diag}\left(\Pi_{1}, I\right)$ has a kernel of dimension $k$, the left-hand sides of the LMI's (38) both have a kernel of dimension $k$ - the LMI's are nonstrict.

Conversely, if (49) vanishes on a common subspace of dimension at least $k$ and if the LMI's (38) are nonstrict but the left-hand sides have a kernel of dimension at most $k$, one can construct a controller of order $n-k$ with (16) as follows: the matrix $I-X Y$ has a kernel of dimension equal to $k$ such that one can find full rank matrices $M, N$ with $k$ columns satisfying $I-X Y=M N^{T}$. We define $\Pi_{1}, \Pi_{2}$ as in (34) and observe that these matrices have full row rank. Since all the blocks in (49) vanish on a common subspace, it is possible to show that there exists a symmetric $\mathcal{P}$ satisfying (34) and (unique) solutions $A_{K}, B_{K}, C_{K}, D_{K}$ of (35). Then, we conclude again that the left-hand sides of (38) and (37) are identical. By assumption, the nonstrict versions of these inequalities hold. With the right-inverse $\Pi_{1}^{+}$of $\Pi_{1}$, we can transform (37) with $\operatorname{diag}\left(\Pi_{1}^{+}, I\right)$ to arrive at the nonstrict inequalities that correspond to (16). Since $\Pi_{1}$ has a kernel of dimension $k$ and since the kernel dimension of the lefthand sides of (37) are at most $k$, it is not difficult to see that the matrices involved in (16) cannot have a kernel at all-the inequalities are strict.

We extract the following conditions for a general synthesis problem: matrices (49) vanish on a common subspace of dimension $k$, and any synthesis LMI is nonstrict such that the matrices involved have a kernel of at most dimension $r k$ if $r$ is the number of matrices $\Pi_{1}$ in the corresponding blockdiagonal congruence transformation.

\section{DESIGN EXAMPLES}

The first example is of academic nature and illustrates the conservatism of this approach. The second example applies the methodology developed in this paper to the design of a dynamic positioning system (DPS) for a moored floating platform. All LMI-related computation was performed with the function hinfmix from the LMI Control Toolbox [18].

Example 7: Consider the three-state unstable plant with equations:

$$
\begin{aligned}
& \dot{x}=\left(\begin{array}{rrr}
0 & 10 & 2 \\
-1 & 1 & 0 \\
0 & 2 & -5
\end{array}\right) x+\left(\begin{array}{l}
1 \\
0 \\
1
\end{array}\right) w+\left(\begin{array}{l}
0 \\
1 \\
0
\end{array}\right) u \\
& y=x_{2}+2 w
\end{aligned}
$$

and performance outputs

$$
z_{\infty}=\left(\begin{array}{c}
x_{1} \\
u
\end{array}\right), \quad z_{2}=\left(\begin{array}{c}
x_{2} \\
x_{3} \\
u
\end{array}\right)
$$

( $u$ and $y$ denote the control and measurement as before). We are interested in the $H_{\infty}$ performance from $w$ to $z_{\infty}$ and the $\mathrm{H}_{2}$ performance from $w$ to $z_{2}$. The optimal $\mathrm{H}_{2}$ performance from $w$ to $z_{2}$ is 7.748 and is achieved for the controller

$$
K_{2}(s)=\frac{-5.7275(s+5.168)(s-0.2711)}{(s+5.164)\left(s^{2}+3.669 s+9.933\right)} .
$$

When closing the loop on $K_{2}(s)$, the $H_{\infty}$ performance from $w$ to $z_{\infty}$ is $\gamma=23.586$.

Now consider the multiobjective problem

$$
\text { Minimize }\left\|T_{w z_{2}}\right\|_{2} \text { subject to }\left\|T_{w z_{\infty}}\right\|_{\infty}<23.6 \text {. }
$$

We know that $K_{2}(s)$ is the optimal solution since it is $\mathrm{H}_{2}$ optimal and satisfies the $H_{\infty}$ constraint. Hence, we can test the performance of our LMI approach to multiobjective design against this known optimal solution. Solving (50) with LMI optimization yields 8.956 as best constrained $H_{2}$ performance, which is $15 \%$ higher than the optimal value 7.748 . The corresponding controller is

$$
K_{\mathrm{LMI}}(s)=\frac{-7.5924(s+5.271)(s-0.05601)}{(s+5.272)\left(s^{2}+4.253 s+10.25\right)}
$$

Note that because we use a common Lyapunov function for both objectives, the LMI-optimal value 8.956 is only an upper bound on the closed-loop $\mathrm{H}_{2}$ performance. In fact, the controller $K_{\mathrm{LMI}}$ has an actual $\mathrm{H}_{2}$ performance of 8.07 (only $4 \%$ above the optimal value 7.748 ) and an $H_{\infty}$ performance of 


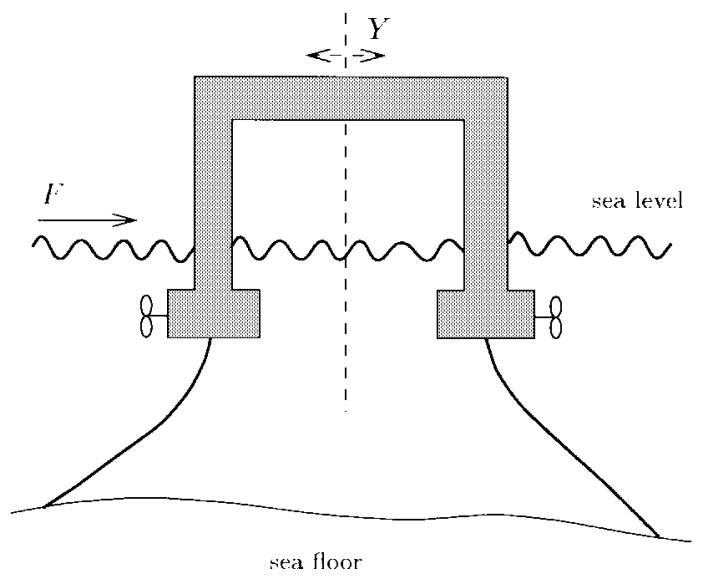

Fig. 1. Moored floating platform.

17.87 [24\% lower than the $H_{\infty}$ performance of the $H_{2}$-optimal controller $K_{2}(s)$ ].

Summing up, this example shows that the LMI approach is indeed conservative, but not unduly so. Meanwhile, it offers the flexibility needed to improve over single-objective "optimal" techniques.

Example 8: This problem was provided by Prof. H. Kajiwara [20], [21]. The system is a floating platform depicted in Fig. 1. This platform is anchored to the bottom of the ocean and equipped with two thrusters. The goal is to minimize the drift $Y$ resulting from the wave action by appropriate thruster control. The wave action can be summarized as a force $F$ and a torque $M$. The force $F$ consists of two components

$$
F=F_{1}+F_{2}
$$

with the following characteristics.

- $F_{1}$ is a high-frequency high-amplitude excitation with small drifting effect. Due to its large magnitude, it cannot be countered by the thrusters.

- $F_{2}$ is a low-frequency low-amplitude excitation that can cause a large drift over time. This drifting action ought to be eliminated by proper thruster control.

The spectral energy of $F_{2}$ is concentrated between 0 and 1 $\mathrm{rd} / \mathrm{s}$, while that of $F_{1}$ is beyond $5 \mathrm{rd} / \mathrm{s}$.

Let $Y, \phi$, and $F_{u}$ denote the horizontal drift, the angular deviation from the vertical axis, and the force delivered by the thrusters, respectively. The control objectives are as follows.

- Reject the drifting force $F_{2}$ by thruster control.

- Maintain $|Y|$ below $0.025 \mathrm{~m}$ and $|\phi|$ below 3 degrees (these figures are for a lab model of the platform).

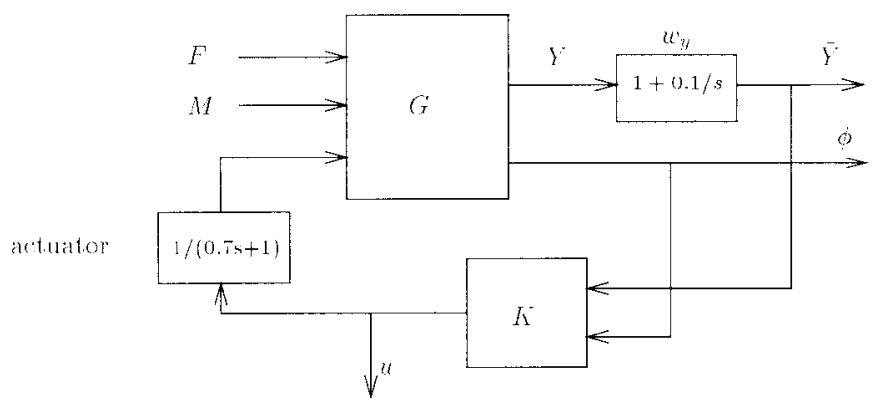

Fig. 2. Control structure.
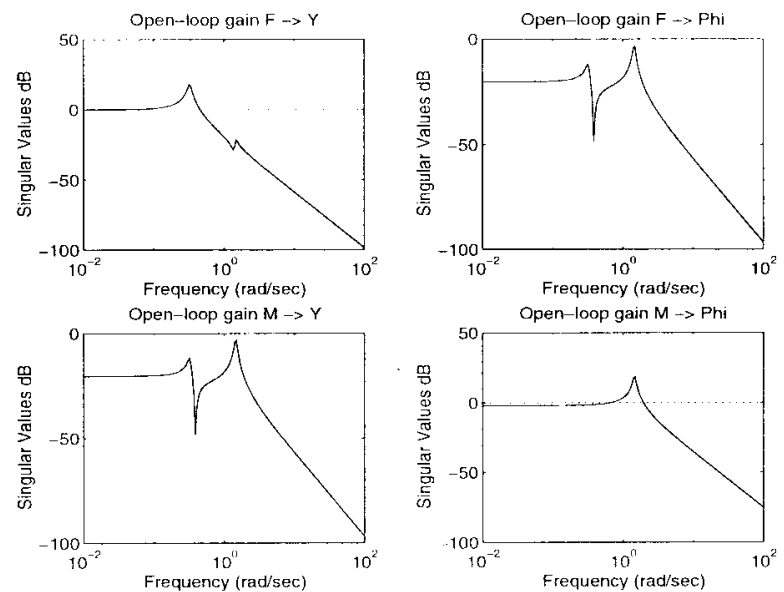

Fig. 3. Open-loop magnitude responses.

- Due to actuator limitations, keep $\left|F_{u}\right|$ below $0.25 \mathrm{~N}$, and make sure that the thrusters are not excited by the high-frequency component $F_{1}$.

Both $Y$ and $\phi$ are measured, and the platform dynamics are described by the state-space equations

$$
\dot{x}=A x+B\left(\begin{array}{c}
F \\
M \\
F_{u}
\end{array}\right), \quad\left(\begin{array}{c}
Y \\
\phi
\end{array}\right)=C x
$$

with $A, B, C$, as shown in (z) at the bottom of the page. The corresponding open-loop frequency responses from $F, M$ to $Y, \phi$ are displayed in Fig. 3. In addition, the thruster dynamics are modeled by the first-order transfer function

$$
F_{u}=d(s) u, \quad d(s)=\frac{1}{0.7 s+1}
$$

where $u$ denotes the control input and $F_{u}$ the actual force delivered by the thrusters; see (z), shown at the bottom of the page.

$$
\left(\begin{array}{c|c}
A & B \\
\hline C & D
\end{array}\right)=\left(\begin{array}{cccc|ccc}
0 & 0 & 1 & 0 & 0 & 0 & 0 \\
0 & 0 & 0 & 1 & 0 & 0 & 0 \\
-0.1010 & -0.1681 & -0.04564 & -0.01075 & 0.1179 & 0.1441 & 0.1476 \\
0.06082 & -2.1407 & -0.05578 & -0.1273 & 0.1441 & 1.7057 & -0.7557 \\
\hline 1 & 0 & 0 & 0 & 0 & 0 & 0 \\
0 & 1 & 0 & 0 & 0 & 0 & 0
\end{array}\right)
$$



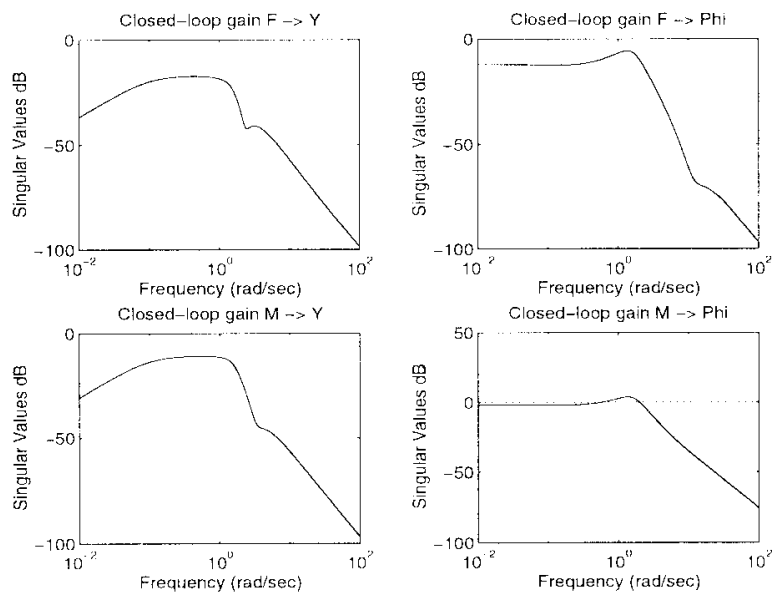

Fig. 4. Design \#1: Closed-loop magnitude responses.
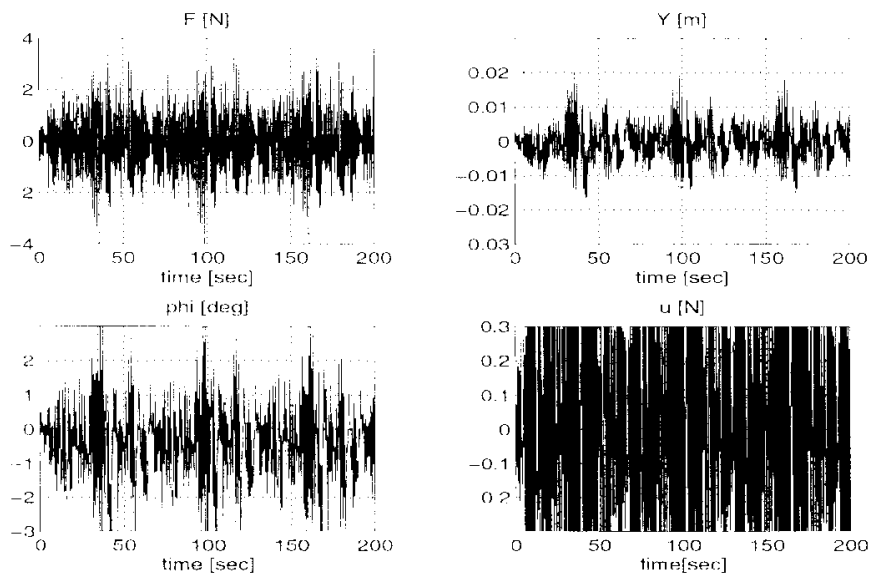

Fig. 5. Design \#1: Closed-loop time responses.

For design purposes, we use the control structure displayed in Fig. 2. Here $K(s)$ denotes the controller to be designed, and the filter $w_{1}(s)=1+0.1 / s$ is used to enforce integral action on the output $Y$. Note that the controller actually implemented would consist of both $K(s)$ and $w_{1}(s)$ (Theorem 5). Our first design is a standard $H_{\infty}$ synthesis aimed at minimizing $\|T\|_{\infty}$, where $T(s)$ is the closed-loop transfer defined by

$$
\left(\begin{array}{c}
\bar{Y} \\
w_{\phi} \phi \\
w_{u} u
\end{array}\right)=T(s)\left(\begin{array}{c}
F \\
M
\end{array}\right), \quad w_{\phi}=w_{u}=0.1 \text {. }
$$

The optimal $H_{\infty}$ performance is approximately 0.4 , and the resulting closed-loop frequency and time responses are shown in Figs. 4 and 5, respectively. The driving force $F$ used in the simulation comes from experimental data over a 200 -s period.

While this $H_{\infty}$ controller meets the rejection specifications on $Y$, it clearly violates the constraint on the maximum thruster effort. To reduce the control effort, we choose to minimize the cost of control in the $H_{2}$ norm while maintaining the $H_{\infty}$ disturbance rejection constraint $\left\|T_{1}\right\|_{\infty}<\underline{0.5}$, where $T_{1}(s)$ is the closed-loop transfer from $\left(\begin{array}{c}F \\ M\end{array}\right)$ to $\left(\begin{array}{c}\bar{Y} \\ w_{\phi} \phi\end{array}\right)$. This is now
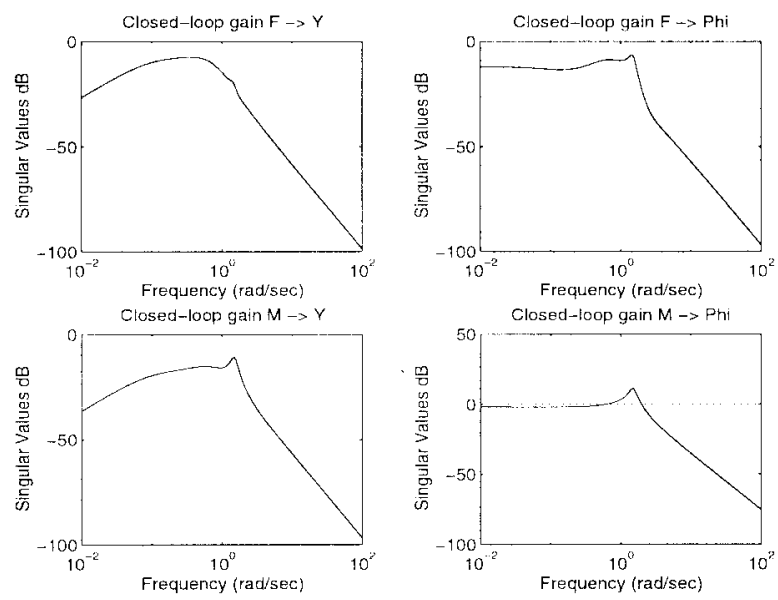

Fig. 6. Design \#2: Closed-loop magnitude responses.
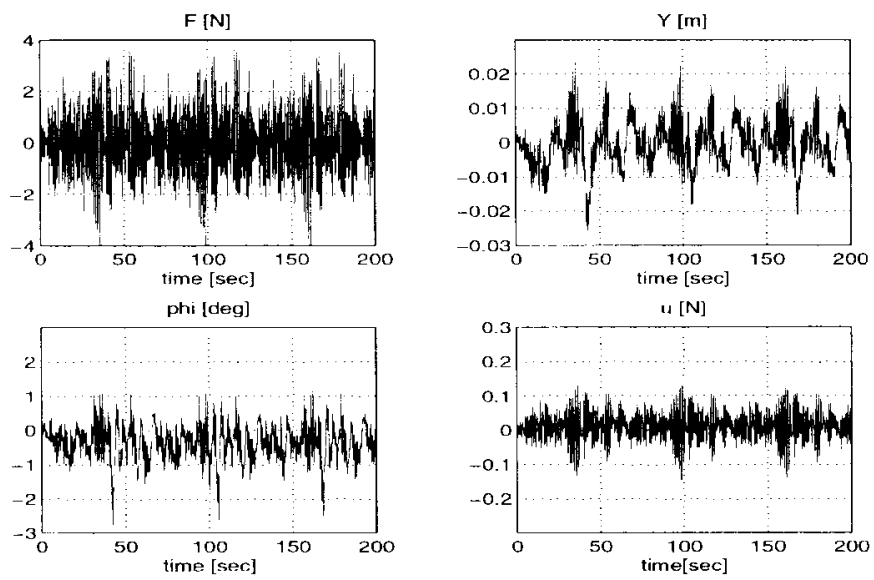

Fig. 7. Design \#2: Closed-loop time responses.
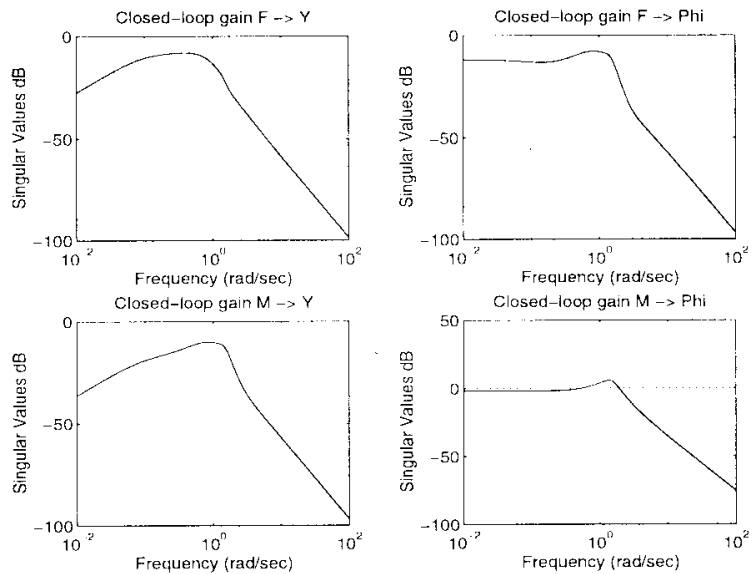

Fig. 8. Design \#3: Closed-loop magnitude responses.

a multiobjective problem with an $H_{\infty}$ constraint on $T_{1}(s)$ and an $H_{2}$ objective on the transfer function $T_{2}(s)$ mapping $\left(\begin{array}{l}F \\ M\end{array}\right)$ to $u$. The corresponding LMI formulation consists of minimizing $\nu$ subject to the synthesis LMI's listed in V-B and V-C. The LMI optimization yields an $\mathrm{H}_{2}$ performance of 1.22 , and the closed-loop frequency and time responses for this second design appear in Figs. 6 and 7. 


$$
\begin{aligned}
& k_{1}(s)=-20.36 \frac{(s+1.43)(s+338.36)\left(s^{2}+254.03 s+3.472 \times 10^{4}\right)\left(s^{2}+0.329 s+3.677 \times 10^{-2}\right)}{s(s+3.25)\left(s^{2}+172.11 s+2.722 \times 10^{4}\right)\left(s^{2}+37.37 s+1.051 \times 10^{3}\right)} \\
& k_{2}(s)=64.37 \frac{(s+1.43)(s+0.23)(s+411.41)\left(s^{2}+51.38 s+2.065 \times 10^{3}\right)}{(s+3.25)\left(s^{2}+172.11 s+2.722 \times 10^{4}\right)\left(s^{2}+37.37 s+1.051 \times 10^{3}\right)}
\end{aligned}
$$

All specifications are now met, and this second design would be deemed satisfactory. Yet, inspection of the closed-loop gain from $M$ to $\phi$ reveals a residual resonance near $1 \mathrm{rd} / \mathrm{s}$ that, if excited, could boost the amplitude of $\phi$. This is confirmed by the presence of a pair of poorly damped modes $-0.168 \pm 1.5 i$ among the closed-loop poles. To dampen these modes, we can resort to an additional regional pole placement objective. For instance, we can impose that the closed-loop modes lie in the conic sector $\mathcal{S}$ with tip at the origin and angle $\theta=6 \pi / 7$. The characteristic function for this LMI region is [11]

$$
f_{\mathcal{S}}(z)=\left(\begin{array}{ll}
\sin \theta(z+\bar{z}) & \cos \theta(z-\bar{z}) \\
\cos \theta(\bar{z}-z) & \sin \theta(z+\bar{z})
\end{array}\right)
$$

To incorporate this additional constraint in the synthesis, we simply add the LMI (45) with $L=0$ and

$$
M=\left(\begin{array}{cc}
\sin \theta & \cos \theta \\
-\cos \theta & \sin \theta
\end{array}\right)
$$

to the system of LMI's used in the second design. The resulting best $\mathrm{H}_{2}$ performance is 2.6, and the closed-loop frequency responses are shown in Fig. 8. The time responses are essentially similar to those for the second design. Note that the resonance has been attenuated, the pair of badly damped modes being moved to $-0.4 \pm 1.46 i$. This final design gives the stable control law $u=k_{1}(s) Y+k_{2}(s) \phi$ as given in the equation shown at the top of the page.

\section{REFERENCES}

[1] J. Abedor, K. Nagpal, P. P. Khargonekar, and K. Poolla, "Robust regulation in the presence of norm-bounded uncertainty," IEEE Trans. Automat. Contr., vol. 40, pp. 147-153, 1995.

[2] J. Abedor, K. Nagpal, and K. Poolla, "Robust regulation with $\mathcal{H}_{2}$ performance," Syst. Contr. Lett., vol. 23, pp. 431-443, 1994.

[3] _ "A linear matrix inequality approach to peak-to-peak gain minimization," Int. J. Robust Nonlinear Contr., vol. 6, pp. 899-927, 1996.

[4] B. D. O. Anderson and S. Vongpanitlerd, Network Analysis. Englewood Cliffs, NJ: Prentice-Hall, 1973.

[5] R. Bambang, E. Shimemura, and K. Uchida, "Mixed $H_{2} / H_{\infty}$ control with pole placement, state-feedback case," in Proc. Amer. Contr. Conf., 1993, pp. 2777-2779.

[6] B. R. Barmish, "Stabilization of uncertain systems via linear control," IEEE Trans. Automat. Contr., vol. AC-28, pp. 848-850, 1983.

[7] D. S. Bernstein and W. M. Haddad, "LQG control with an $H_{\infty}$ performance bound: A Riccati equation approach," IEEE Trans. Automat. Contr., vol. 34, pp. 293-305, 1989.

[8] S. P. Boyd and L. El Ghaoui, "Method of centers for minimizing generalized eigenvalues," Lin. Alg. Appl., vol. 188, pp. 63-111, 1993.

[9] S. Boyd, L. El Ghaoui, E. Feron, and V. Balakrishnan, Linear Matrix Inequalities in Systems and Control Theory. Philadelphia, PA: SIAM, 1994.
[10] M. K. K. Cevik and J. M. Schumacher, "The regulator problem with robust stability," Rep. BS-R9321, CWI, Amsterdam, 1993.

[11] M. Chilali and P. Gahinet, " $H_{\infty}$ design with pole placement constraints: An LMI approach," IEEE Trans. Automat. Contr., vol. 41, pp. 358-367, 1996.

[12] E. J. Davison, "The robust control of a servomechanism problem for linear time-invariant multivariable systems," IEEE Trans. Automat. Contr., vol. AC-21, pp. 25-34, 1976.

[13] J. C. Doyle, K. Zhou, K. Glover, and B. Bodenheimer, "Mixed $\mathcal{H}_{2}$ and $\mathcal{H}_{\infty}$ performances objectives II: Optimal control," IEEE Trans. Automat. Contr. vol. 39, pp. 1575-1587, 1994.

[14] B. A. Francis, "The linear multivariable regulator problem," SIAM J. Contr. Optim., vol. 15, pp. 486-505, 1977.

[15] _ A Course in $H_{\infty}$ Control Theory, Lecture Notes in Control and Information Sciences, no. 88. Berlin: Springer-Verlag, 1987.

[16] P. Gahinet and P. Apkarian, "A linear matrix inequality approach to $H_{\infty}$ control," Int. J. Robust Nonlinear Contr., vol. 4, pp. 421-448, 1994.

[17] P. Gahinet, "Explicit controller formulas for LMI-based $H_{\infty}$ synthesis," Automatica, to appear.

[18] P. Gahinet, A. Nemirovski, A. J. Laub, and M. Chilali, LMI Control Toolbox. Natick, MA: The MathWorks, 1995.

[19] T. Iwasaki and R. E. Skelton, "All controllers for the general $\mathcal{H}_{\infty}$ control problem: LMI existence conditions and state space formulas," Automatica, vol. 30, pp. 1307-1317, 1994.

[20] M. Nakamura, W. Koterayama, H. Kajiwara, and T. Mitamura, "Application of a dynamic positioning system to a moored floating platform," in Proc. 4th Int. Offshore Polar Eng. Conf., 1994, pp. 190-197.

[21] H. Kajiwara, W. Koterayama, M. Nakamura, and P. Gahinet, "LMIbased design of dynamic positioning control laws for a moored floating platform model," in Proc. 14th SICE Kyushu Branch Annu. Conf., 1995, pp. 181-184.

[22] P. P. Khargonekar and M. A. Rotea, "Mixed $H_{2} / H_{\infty}$ control: A convex optimization approach," IEEE Trans. Automat. Contr., vol. 39 , pp. 824-837, 1991.

[23] I. Masubuchi, N. Suda, and A. Ohara, "LMI-based controller synthesis: A unified formulation and solution," in Proc. Amer. Contr. Conf., 1995, pp. 3473-3477.

[24] I. Masubuchi, A. Ohara, and N. Suda, "LMI-based controller synthesis: A unified formulation and solution," Int. J. Robust Nonlinear Contr., submitted.

[25] A. Nemirovski and P. Gahinet, "The projective method for solving linear matrix inequalities," Math. Programming Series B, to be published; also in Proc. Amer. Contr. Conf., 1994, pp. 840-844.

[26] Y. Nesterov and A. Nemirovski, Interior Point Polynomial Methods in Convex Programming: Theory and Applications. Philadelphia, PA: SIAM, 1994.

[27] M. A. Rotea, "The generalized $\mathcal{H}_{2}$ control problem," Automatica, vol. 29, pp. 373-385, 1993.

[28] C. Scherer, "The Riccati inequality and state-space $H_{\infty}$-optimal control," Ph.D. dissertation, Univ. Würzburg, Germany, 1990.

[29] _ "Mixed $H_{2} / H_{\infty}$ control," in Trends in Control, A European Perspective, A. Isidori, Ed. Berlin: Springer-Verlag, 1995, pp. $173-216$.

[30] _ "From LMI analysis to multichannel mixed LMI synthesis: A general procedure," Selected Topics Identification, Modeling Contr., vol. 8, pp. $1-8,1995$.

[31] W. Sun, P. P. Khargonekar, and D. Shim, "Solution to the positive real control problem for linear time-invariant systems," IEEE Trans. Automat. Contr., vol. 39, pp. 2034-2046, 1994.

[32] L. Vandenberghe and S. Boyd, "Primal-dual potential reduction method for problems involving matrix inequalities," Math. Program. Series B, vol. 69 , pp. $205-236,1995$.

[33] J. C. Willems, "Least-squares stationary optimal control and the algebraic Riccati equation," IEEE Trans. Automat. Contr., vol. AC-21, pp. 319-338, 1971. 


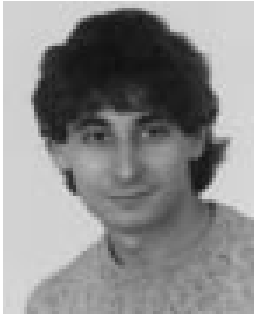

Carsten Scherer (M'94) was born in Lohr, Germany. He received the diploma and the Ph.D. degree in mathematics from the University of Würzburg, Germany, in 1987 and 1991, respectively.

He was appointed a Research Assistant from 1988 until 1993 at University of Würzburg, Germany. In 1989, he visited the Mathematics Institute of the University of Groningen, The Netherlands, for six months. In 1992, he received a Post-Doctoral Fellowship from the "Deutsche Forschungsgemeinschaft" (DFG) to spend six months at the University of Michigan, Ann Arbor, and six months at Washington University, St. Louis, MO, respectively. In 1993, he joined the Mechanical Engineering Systems and Control Group at Delft University of Technology, The Netherlands, where he currently holds a position as Assistant Professor ("Universitair Docent"). His research interests include robust control for linear and nonlinear systems, geometric control, the theory of linear matrix inequalities, Riccati inequalities, and Riccati equations in control.

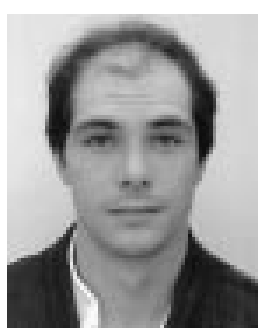

Pascal Gahinet (M'90) graduated from the Ecole Polytechnique in 1984 and from the ENSTA, Paris, France, in 1986, and received the M.S. degree in electrical and computer engineering and the Ph.D. degree in system theory, both from the University of California, Santa Barbara, in 1987 and 1989, respectively.

He was with the French National Research Institute in Computer and Control Sciences (INRIA) from 1990 to 1995, taught control at ENSTA from 1991 to 1994, and also served as a Consultant for THOMSON-CSF and Aérospatiale during this period. He is now with The MathWorks, Inc., Natick, MA. His research interests include robust control theory, linear matrix inequalities, numerical linear algebra, and numerical software for control. He is a coauthor of the LMI Control Toolbox and the Control System Toolbox for use with MATLAB.

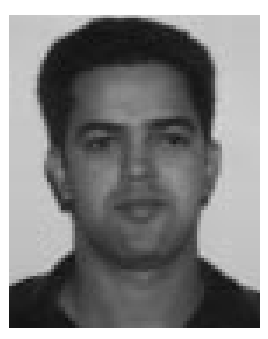

Mahmoud Chilali was born in Casablanca, Morocco, in 1966. He received the engineer diplomas from the Ecole Polytechnique and the Ecole $\mathrm{Na}$ tionale Supérieure de Techniques Avancées, Paris, France, in 1990 and 1992, respectively, and the Ph.D. degree from the French Institut National de Recherche en Informatique et Automatique (INRIA) in 1996.

His research interests include robust and multiobjective control and LMI optimization. 